\title{
Focusing of an elongated hole in porous medium
}

\author{
flow \\ S. B. Angenent \\ Mathematics Department, University of Wisconsin, Madison \\ D. G. Aronson, S. I. Betelu and J. S. Lowengrub \\ Mathematics Department, University of Minnesota \\ 127 Vincent Hall, 206 Church St SE, Minneapolis, MN 55455 \\ betelu@math.umn.edu, FAX 612-626-7370
}

\begin{abstract}
In the focusing problem we study solutions to the porous medium equation $\partial_{t} u=\Delta\left(u^{m}\right)$ whose initial distributions are positive in the exterior of a compact two-dimensional region and zero inside. We assume that the initial interface is elongated (i.e., has an aspect ratio $>1$ ), and possesses reflectional symmetry with respect to both the $x$ - and $y$-axes. We implement a numerical scheme that adapts the numerical grid around the interface so as to maintain a high resolution as the interface shrinks to a point. We find that as $t$ tends to the focusing time $T$, the interface
\end{abstract}


becomes oval-like with lengths of the major and minor axes $O(\sqrt{T-t})$ and $O(T-t)$ respectively. Thus the aspect ratio is $O(1 / \sqrt{T-t})$. By scaling and formal asymptotic arguments we derive an approximate solution which is valid for all $m$. This approximation indicates that the numerically observed power law behavior for the major and minor axes is universal for all $m>1$.

PACS: 02.70.-c; 05.10.Cc; 05.10.-a; 47.55.Mh

Keywords: Porous medium flow; Similarity; Self-similarity; Diffusion; Nonlinear

\section{Introduction}

This work concerns convergent flows governed by the nonlinear degenerate diffusion equation

$$
\frac{\partial u}{\partial t}=\Delta\left(u^{m}\right)
$$

where $m>1$ is a constant and $\Delta$ denotes the Laplace operator in $d$-dimensional Euclidean space $\mathbf{R}^{d}$. Equation (1) models the evolution of the density of an ideal gas flowing isentropically in a homogeneous porous medium $[5,8]$ and is usually called the porous medium equation. However, it also arises in various other applications such as population biology [21], diffusion of strong thermal waves [9], and, most notably for our purposes, the spreading of viscous gravity currents $[19,12,13]$. We are interested in the focusing problem for equation (1) in which we study flows which start at time $t=0$ from an initial distribution which is 
concentrated outside some compact set $\mathbf{K}$ in $\mathbf{R}^{d}$. Because of the degeneracy of the diffusion coefficient in equation (1), material flows into the empty region $\mathbf{K}$ with a finite speed of propagation, and eventually first covers all of $\mathbf{K}$ at some finite time $T>0$, called the focusing time. The boundary between the empty and full regions at any given time is called the interface. We are particularly interested in the final shape of the interface, i.e., its shape at times just before the focusing time.

By Darcy's law, the velocity in porous medium flow is proportional to the pressure gradient. Thus, in order to study the evolution of the interface it is convenient to replace the density $u$ as the independent variable equation (1) with the (scaled) pressure

$$
v \equiv \frac{m}{m-1} u^{m-1}
$$

The equation satisfied by $v$ is the porous medium pressure equation

$$
\frac{\partial v}{\partial t}=(m-1) v \Delta v+|\nabla v|^{2}
$$

In terms of the pressure, the local velocity of the interface $v=0$ is given by

$$
\mathbf{U}=-\nabla v
$$

From the numerical point of view the pressure equation Eq. (3) is more convenient than Eq. (1) because the gradient of $v$ is bounded at the interface, while the gradient of $u$ may either tend to zero or diverge, depending on $m$.

We will restrict our attention to flows in the plane $\mathbf{R}^{2}$. The case in which the empty region $\mathbf{K}$ is a circle is well-studied experimentally, numerically and theoretically. To begin with, there exists a one parameter family of axially 
symmetric self-similar solutions (the Graveleau solutions $[6,17]$ ) which describe, to leading order, the focusing of essentially any axially symmetric flow $[1,2]$. In particular, the radius $R$ of the shrinking interface obeys a power law of the form $R \sim(T-t)^{\beta}$, where $T$ is the focusing time and $\beta$ is an exponent which depends on the parameter $m$. This power law behavior has been observed experimentally and verified numerically for viscous gravity current flows in the regime where capillarity is negligible [12]. Such flows are modeled by Eq. (1) with $m=4$. Additional experimental and numerical studies [13] indicate that if the region $\mathbf{K}$ has an elongated shape (e.g., is an ellipse) then the final shape is not circular, but is instead some sort of non-self-similar oval whose major and minor axes obey different power laws.

The observations for the non-axial case were confirmed by a linearized stability analysis of the axially symmetric interfaces for the Graveleau solutions [11]. That analysis shows that the Graveleau interfaces are unstable with respect to perturbations with wave number $k=2$ for an interval of values of $m>1$ [11]. For wave numbers $k>2$, the situation is more complicated. For each integer $k>2$ there exist an $m_{k}$ such that

$$
\infty>m_{3}>m_{4}>\cdots>m_{k}>m_{k+1}>\cdots \searrow 1
$$

and the Graveleau interfaces are stable with respect to perturbations with wave number $k$ for $m>m_{k}$ and unstable with respect to such perturbations for $m<m_{k}$. In [3] it is shown that there is a bifurcation to a branch of self-similar solutions with $k$-fold symmetry at each $m=m_{k}$, at least for sufficiently large $k$. The self-similarity exponents on the new branches depend on $m$ and $k$. The 
bifurcation diagram is computed in [11], without restriction to large $k$, along with the form of the bifurcating solutions. In this paper we study the evolution of initially elongated empty regions $\mathbf{K}$. The specific form of the elongated empty region is not critical, so for simplicity we restrict our attention to the case in which $\mathbf{K}$ is an ellipse. Using an extension of the renormalization technique introduced in [11] (a numerical scheme that rescales the numerical grid and the solution so as to maintain a high resolution near the focusing time) together with an adaptation the Osher-Sethian level set method for tracking the interface [26], we perform very detailed and accurate numerical integration of the initial value problem for Eq. (3). From this we observe that the shrinking interface tends to an oval whose major axis has length $x_{f} \sim \sqrt{T-t}$ and whose minor axis has length $y_{f} \sim T-t$ as $t \nearrow T$. Thus the aspect ratio of the interface (i.e., the ratio of the lengths of the major and minor axes) is $O\left((T-t)^{-\frac{1}{2}}\right)$ as $t \nearrow T$. The number of renormalization steps needed in order to reach the asymptotic regime increases rapidly as the parameter $m$ is increased. Consequently for values of $m>6$ we are unable to maintain sufficient accuracy in our front tracking to be able to obtain reliable values of the lengths of the major and minor axis. The increase of renormalization steps needed is to be expected because the radially symmetric Graveleau solutions become neutrally stable with respect to elongating perturbations as $m$ tends to infinity (see Appendix 2 and [18]). In that limit the focusing problem for the porous medium pressure equation 
becomes the Hele-Shaw problem

$$
\begin{aligned}
& \Delta v=0 \quad \text { on } P(t) \\
& v=0 \text { and } \frac{\partial v}{\partial t}=|\nabla v|^{2} \text { on } \partial P(t),
\end{aligned}
$$

where $P(t)=\{(x, t): v(x, t)>0\}$, and the Graveleau solution converges to a self-similar solution to (6) with similarity exponent $\beta=1 / 2$ [7]. However, the self-similar solutions to (6) with radial interface are embedded in a family of selfsimilar solutions with elliptic interfaces, all with similarity exponent $\beta=1 / 2$. For these solutions the aspect ratio is constant.

In the limit $m=1$, the focusing problem for Eq. (3) becomes the focusing problem for the Hamilton-Jacobi equation

$$
\frac{\partial v}{\partial t}=|\nabla v|^{2}
$$

[20]. The viscosity solutions of the Hamilton-Jacobi equation are given in terms of the initial data $v(x, 0)=v_{0}(x)$ by the Lax-Oleinik formula

$$
v(x, t)=\sup _{y \in \mathbf{R}^{2}}\left\{v_{0}(y)-\frac{|x-y|^{2}}{4 t}\right\}
$$

From this expression one sees by elementary arguments that focussing for a typical solution to Eq. (7) can proceed along one of two different courses. In the first case the solution is locally given by three traveling plane waves which converge to one point. In this case the interface just before focusing has the shape of a triangle. In the other case the solution is given by two non-planar traveling waves. The non-flatness of the waves causes the interface to have the shape of two parabolae which pass through each other at the focusing time. 
This latter solution is asymptotically given by

$$
v(x, y, t)=A(t-T)+x^{2}+|y|+\text { smaller terms }
$$

We refer to it as the "closing eye solution."

A noteworthy feature of our numerical studies is that, at least for $m \in[1,6]$, the power law behavior of the shrinking interfaces is independent of the value of $m$. We conjecture that this remains true for all values of $m$. In support of this conjecture we construct a formal asymptotic approximate solution to Eq. (3) which is valid for any value of $m \geq 1$. This approximation has a discontinuous derivative at the apex of the interface on the major axis, but otherwise very closely matches the numerical results.

We also present a formal scaling argument which identifies our asymptotic approximation as the correct limit at the focusing time, and thus lends further support to our conjecture about the universality of the interface power laws with respect to $m$.

The outline of this paper is as follows: in Section 2 we describe the numerical method used to integrate Eq. (3) and the renormalization technique needed to accurately describe the shape of the interface as its size tends to zero. There we also present the benchmark problems used to validate the numerics. In Section 3 we present the results on the focusing of an elongated cavity. In Section 4 we discuss a heuristic analytic approximation of the asymptotic regime, and in Section 5 we study the same approximation with a more formal asymptotic analysis. In Appendix 1 we describe, in detail, the numerical computations of derivatives, while in Appendix 2 we describe the stability calculations for the 
Graveleau interface for very large values of $m$. In Appendix 3 we sketch the proofs of some of the properties of our asymptotic approximation.

\section{Numerical procedure}

Here we describe the procedure used to find numerical asymptotic solutions. To solve Eq. (3) numerically, we discretize a rectangular domain $D=\left[0, L_{x}\right] \times$ $\left[0, L_{y}\right]$ with a uniform grid of interval sizes $\Delta x=L_{x} / N$ and $\Delta y=L_{y} / N$ (see Fig. (1)). The solution is stored in a matrix as $v\left(x_{i}, y_{j}, t\right)=v_{i j}$ with $x_{i}=i \Delta x$ and $y_{j}=j \Delta y$. In order to integrate the PDE in time, we use an explicit Euler scheme

$$
v_{i j}(t+\Delta t)=v_{i j}(t)+\Delta t H_{i j}
$$

where $H_{i j}$ is a finite differences approximation of the derivatives of the right hand side of Eq. (3). We compute the derivatives of the term $|\nabla v|^{2}=v_{x}^{2}+v_{y}^{2}$ with a second order upwind ENO (essentially non-oscillatory) scheme [25, 23] (see Appendix 1). This method guarantees that the numerical scheme will be able to accurately describe the discontinuities in the first derivative that spontaneously appear in the case $m=1$ (Hamilton-Jacobi limit) and at the interface. The derivatives of the Laplacian term in Eq. (3) are computed with standard centered second order differences,

$$
\begin{gathered}
\Delta v=v_{x x}+v_{y y} \simeq \\
\simeq \frac{v_{i+1, j}-2 v_{i j}+v_{i-1, j}}{(\Delta x)^{2}}+\frac{v_{i, j+1}-2 v_{i j}+v_{i, j-1}}{(\Delta y)^{2}} .
\end{gathered}
$$


At the interface there is a discontinuity in the first derivative that may generate numerical errors when we compute the Laplacian with Eq. (10). When a grid point $v_{i-1, j}$ is situated inside the hole and adjacent to the interface, $v_{x x}$ is computed with a quadratic fit of the positive values of $v$ at the points $v_{i, j}, v_{i+1, j}$ and $v_{i+2, j}$. When $v_{i, j-1}$ is situated inside the hole, $v_{y y}$ is computed with $v_{i, j}, v_{i, j+1}$ and $v_{i, j+2}$ (see Fig. (2)). We use Eq. (8) only outside the hole, thus we don't need to consider the case when $v_{i j}$ is inside the interface. We come back to this issue in 2.1 , where we explain in detail how we detect the interface.

At the boundaries $x=0, y=0$ we apply the boundary conditions of symme$\operatorname{try} v_{x}=0$ and $v_{y}=0$, respectively. Since we are looking for a focusing solution that extends to infinity, we put the outer walls of the grid far from the interface $\left(L_{x} \approx 7 x_{f}, L_{y} \approx 7 y_{f}\right)$, and at these boundaries $x=L_{x}$ and $y=L_{y}$ we apply the boundary condition $v_{x x}=0$ and $v_{y y}=0$, which is equivalent to a first order

linear extrapolation of $v$ outside $D$. The comparison with exact solutions (see 2.4) shows that this approximation works very well.

\subsection{Representation of the interface}

In order to accurately describe the shape of the interface we use a level set representation $[26,22]$. We define a function $\phi$ which has the following properties:

- $\phi(x, y, t)>0$ in the region occupied by the fluid (where $v>0$ ).

- $\phi(x, y, t)=0$ at the interface.

- $\phi(x, y, t)<0$ inside the interface (the hole). 
(See Fig. (1)). The idea behind the level set method is to move the contour line $\phi=0$ with the correct speed $\mathbf{U}=-\nabla v$ and to compute the pressure $v$ taking into account the position of the interface stored implicitly in $\phi$. One convenient choice for $\phi$ is a function that satisfies

$$
\frac{\partial \phi}{\partial t}=|\nabla \phi|^{2} \quad \phi<0 \quad \text { inside the interface }
$$

and

$$
\phi(x, y, t)=v(x, y, t) \geq 0 . \quad \text { in the fluid region }
$$

In other words, the level lines of $\phi$ move with a normal velocity given by $|\nabla \phi|$. In particular, if we require that the function $\phi$ has the same normal derivative as $v$ at the interface, then the interface is going to move at the velocity given by Eq. (4).

In our numerical scheme we solve Eq. (3) together with Eq. (11) as

$$
\phi_{i j}(t+\Delta t)=\phi_{i j}(t)+\Delta t F_{i j} \quad \text { for } \phi_{i j}<0
$$

where $F_{i j}$ are the discretized derivatives of $|\nabla \phi|^{2}=\phi_{x}^{2}+\phi_{y}^{2}$ computed with a second order upwind ENO scheme. For $\phi_{i j} \geq 0$ we simply put $\phi_{i j}=v_{i j}$.

The boundary condition for $\phi$ at the interface is

$$
\frac{\partial \phi}{\partial n}=\frac{\partial v}{\partial n}+\quad \text { at } v=0
$$

where $n$ denotes the normal to the interface. This condition is satisfied automatically since we compute the derivatives of $\phi$ with an upwind approximation, and near the interface they are computed with the values of $\phi>0$, which are equal to $v$ (see Fig. (2)). When we compute the derivatives of the pressure $v$, we 
do not use the values inside the hole because we have upwind approximations. In other words, the function $v$ does not directly 'see' the function $\phi$. The only information from $\phi$ that we use is the position of the interface.

We find that the contours of $\phi$ do not cluster, so that no re-distancing [24] is necessary for $\phi$. (Roughly speaking, this is because Eq. (11) is the natural continuation of Eq. (3) at $v=0$.)

To avoid instabilities due to the diffusive term, the time step must satisfy a stability condition

$$
\Delta t<\Delta t_{0}=\min \frac{(\Delta y)^{2}}{2(m-1) v_{i j}}
$$

where the minimum is computed with all the points belonging to the region of the fluid $(v>0)$. (Here we are using the fact that we always make the computations with $\Delta y \leq \Delta x)$. The $|\nabla v|^{2}$ term in Eq. (3) and $|\nabla \phi|^{2}$ in Eq. (11) are less restrictive, and must satisfy

$$
\Delta t<\Delta t_{1}=\min \left(\min \left(\frac{\Delta x}{\left|\phi_{x}\right|}, \frac{\Delta y}{\left|\phi_{y}\right|}\right)\right),
$$

where the first minimum is computed with all the points of the domain.

In order to increase the accuracy of the computation near the interface, we take into account an important property of the solution: if at any point $v=0$, then $v_{t}=0$ unless the interface is exactly at this point. We shall enforce this property in our numerical solution in the following way:

$$
\text { if } \quad v_{i j}=0 \text { and } \phi_{i j}<0 \text { then } H_{i j}=0
$$

and we start to update $v$ with Eq. (8) only when the interface crosses the point $\left(x_{i}, y_{j}\right)$, as shown in Fig. (3) (i.e. when $\left.\phi_{i j} \geq 0\right)$. We also must avoid having 
the interface 'jump' over a grid point in a time interval $\Delta t$. We can avoid the interface jumping a grid point and at the same time satisfy Eqs. $(15,16)$ if we compute the time-step as

$$
\Delta t=\min \left(\Delta t_{0}, \Delta t_{1}, \frac{-\phi_{i j}}{F_{i j}}\right)
$$

The minimum is computed with all the points adjacent to the interface where $\phi_{i j}<0$. When the third argument is the smallest, the interface will coincide exactly at the gridpoint $\left(x_{i}, y_{j}\right)$.

\section{$2.2 \quad$ Initial conditions}

We start the integration with a rather arbitrary initial condition,

$$
v^{(0)}\left(x, y, t_{0}\right)=\sqrt{\frac{x^{2}}{a^{2}}+\frac{y^{2}}{b^{2}}}-1
$$

that describes a function with elliptical contour lines. In the results reported here, we use $a=0.22, b=0.20$ and $L_{x}=L_{y}=1$. The exact form of the initial condition is not very critical, because the asymptotic solution only depends on $m$, as was verified numerically. For instance, later we shall show that we get the same results with an initial condition where the contour lines of $v$ are rectangular.

\subsection{Numerical renormalization}

The numerical renormalization method was inspired by $[16,15]$ and first applied in this context in [11]. A related method was also used in [10]. We start with the initial condition stored in a square grid $L_{y}=L_{x}$. At time $t_{n}$ (initially 
$n=0)$ we start to integrate the diffusion equation until the major axis of the interface reaches a half of its initial value at time $t_{n+1}$, and then we renormalize the solution in the following way [11]

$$
v^{(n+1)}\left(x, y, t_{n+1}\right)=Z^{(n)} v^{(n)}\left(x / 2, y / 2, t_{n+1}\right) .
$$

This transformation is performed by quadratically interpolating the grid values. First we interpolate in the $x$ direction,

$$
v_{i j}^{(n+1)}=Z^{(n)} v_{i / 2, j}^{(n)} \quad \text { for even } i
$$

and

$$
v_{i j}^{(n+1)}=Z^{(n)}\left(3 v_{i / 2+1, j}^{(n)}+6 v_{i / 2, j}^{(n)}-v_{i / 2-1, j}^{(n)}\right) / 8 \quad \text { for odd } i
$$

and then we repeat the analogous procedure in the $y$ direction. The constant $Z^{(n)}$ is taken as the inverse of the maximum value of the function $v$ in the renormalized domain of integration

$$
Z^{(n)}=\left(\max v^{(n)}\left(x, y, t_{n+1}\right)\right)^{-1}
$$

The superscript $(n)$ indicates how many renormalizations we have made up to the given time. The errors introduced in the interpolation are of third order.

We continue integrating the diffusion equation and making the renormalization each time that the major axis of the interface reaches a half of its initial value.

If the initial condition produces a self-similar focusing solution (for example if it has circular symmetry), then the numerical solution will converge to the 
self-similar solution (see 2.4 and [11]). However, here we are interested in the case where we do not have a self-similar solution. In this case the interface may become very elongated and the discretization may eventually not be enough to accurately describe the solution (see Fig. (4)). In order to overcome this problem, we adjust the aspect ratio of the integration domain $D$ to be similar to the aspect ratio of the interface. Specifically, we divide the $y$-dimension of the integration domain by a factor two when the aspect ratio of the interface increases by a factor two. In this way we maintain a good discretization around the interface. In fact we keep the size of the domain of integration of the order of $L_{x} \sim 7 x_{f}$ and $L_{y} \sim 7 y_{f}$. After dividing the integration domain $M$ times, the dimensions will be

$$
D=\left[0, L_{x}\right] \times\left[0,2^{-M} L_{x}\right]
$$

where we have $L_{x}=L_{y}$ at the beginning of the computation.

In order to compute the exponents $\alpha_{x}$ and $\alpha_{y}$ for the shrinking semiaxis, we fit a power law of the form

$$
x_{f}(t)=A(T-t)^{\alpha_{x}}
$$

to three consecutive values of $x_{f}$ sampled at the renormalization time (each time the major axis reduces by a half). The analogous procedure is done to compute $\alpha_{y}$, where the three values of $y_{f}$ are sampled at the time when the grid is divided in the $y$ direction (each time the aspect ratio increases by a factor two). 


\section{$2.4 \quad$ Validation}

1. In order to show the accuracy of this numerical computation, we compare in Table I the numerical similarity exponents $\alpha_{x}$ for the circularly symmetric solution with the exact Graveleau exponents [6], and we find a good agreement ${ }^{1}$.

$\begin{array}{ccc}m & \text { Numeric } & \text { 'Exact' } \\ 1.5 & 0.90944 & 0.90898 \\ 2.0 & 0.85657 & 0.85633 \\ 2.5 & 0.82123 & 0.82145 \\ 3.0 & 0.79588 & 0.79634 \\ 3.5 & 0.77659 & 0.77724 \\ 4.0 & 0.76131 & 0.76203\end{array}$

Table I: Exponents for the circular case, $L_{x}=L_{y}=1, a=b=0.1$ and

$$
N=200
$$

This comparison checks the accuracy of the renormalization procedure and the temporal integration.

2. We also computed the solutions corresponding to the Hamilton Jacobi case $m=1$ without circular symmetry, and we compared the numerical

\footnotetext{
${ }^{1}$ We implemented the numerical scheme in FORTRAN language and we made the computations in an Indigo Silicon Graphics computer (R10000-195 MHZ processor)
} 
result with the exact solution

$$
v(x, y, t)=\frac{1}{8}\left(4(|y|+t)-1+\sqrt{16 x^{2}+8(|y|+t)+16(|y|+t)^{2}+1}\right) .
$$

In Fig. (5) we show a contour plot of this solution. This solution, the so called "closing-eye" solution, is particularly important here, since we can use it as an initial condition, and then we can check the value of the numerical exponents and collapse time. The interfaces of this exact solution are two arcs of parabola, and the exponents of the major and minor axis are exactly $\alpha_{x}=1 / 2$ and $\alpha_{y}=1$, respectively. The relative departure of the numerical solution from the exact solution is the cumulative error

$$
\Delta=\frac{1}{v_{\max }} \sqrt{\frac{1}{L_{x} L_{y}} \iint\left|v^{(n)}\left(x, y, t_{n}\right)-v\left(x, y, t_{n}\right)\right|^{2} d x d y}
$$

where $v$ is computed with Eq. (26) and $v_{\max }$ is the maximum value of the solution in $\left[0, L_{x}\right] \times\left[0, L_{y}\right]$. We compute this cumulative error with the initial condition given by Eq. (26) with $t_{0}=-1 / 16, x_{f}=1 / 4$ and $y_{f}=1 / 16$. Then we integrate numerically with 100 gridpoints and we obtain an absolute error in the focusing time $T$ of less than $10^{-6}$. The absolute error of the numerical exponents is smaller than $10^{-5}$. In Fig. (6) we show the cumulative error as a function of the number of gridpoints $N$, after 4 renormalizations and 5 regriddings (at this point, the value of $x_{f}$ decreased in a factor 16 , and the aspect ratio is $x_{f} / y_{f}=64$. The slope of the points in this graph is close to -2 , indicating that the numerical scheme has quadratic convergence. 
This comparison checks the accuracy of the two-dimensional evaluation of the $|\nabla v|^{2}$ term, the renormalization procedure and the dynamic regridding.

\section{Numerical results}

In Fig. (7) we show the contour lines of $v$ for an initial condition with elliptical interfaces of semiaxis $a=0.22$ and $b=0.20$ and $m=2$. We plotted the contour lines of the initial condition, and after 2, 4, and 6 renormalizations. Here we did not regrid the $y$-direction to show the increasing elongation. We discretized the domain with $100 \times 100$ points.

In Fig. (8) we show the contour lines of $v$ for the same initial condition. We plotted the contour lines of the initial condition, and after 2,4 , and 7 renormalizations and rescaling $x$ and $y$ with the major and minor axis, respectively. Here we apply the regridding in the $y$-direction to maintain a good description of the interface.

We investigated various initial conditions, and from this we observed that the asymptotic shape of the contour lines is qualitatively insensitive to the initial condition, provided there is symmetry respect to the $x$ - and $y$-axis and elongation. This is in agreement with the experimental results in [13]. For example, in Fig. (9) the initial condition has rectangular equi-spaced contour lines, and the initial interface is a rectangle of dimensions $0.22 \times 0.20$. As the aspect ratio increases, the shape of the contour lines approach those of Fig. 
(8). We observe the same results for circularly symmetric initial conditions perturbed with small elongations.

Moreover, for a fixed $m$, as $x_{f} / y_{f} \rightarrow \infty$, the shape of the contour lines of the asymptotic solution converge to a universal picture by rescaling the $x$-coordinate and the $y$-coordinate with $x_{f}$ and $y_{f}$, respectively. The shape of the interfaces are approximately equal to two opposing arcs of parabolas. Solutions of the porous medium equations have smooth interfaces, but the numerical simulation cannot resolve the rounding of the tip because the radius of curvature is much smaller than the discretization. We conjecture that the size $R$ of this curvature radius tends to zero when rescaled with $x_{f}$, i.e. $R / x_{f} \rightarrow 0$ as $T-t \rightarrow 0$. In Fig. (10) we show the contour lines of the numerical solution for $m=2$ and $m=4$ for $x_{f} / y_{f} \approx 1000$.

In Fig. (11) we show the major and minor axis as a function of the time, as measured from the focusing time $T-t$. Note that our numerical procedure can describe 12 orders of magnitude on the time scale. Asymptotically as $T-t \rightarrow 0$ the axes follow power laws on the form $x_{f} \sim(T-t)^{1 / 2}$ and $y_{f} \sim T-t$. The errors on the determination of these exponents are of the order of $10^{-5}$, which is the amplitude of the numerical variations of the values obtained from the computation. These power laws are the same for $1 \leq m \leq 6$.

One important question that we can formulate is the following: how fast does the hole elongate?. We performed a computation to answer the more restricted question: if the initial hole has an aspect ratio of 1.1, how much does the 
hole have to shrink until the aspect ratio reaches 10? In Fig. (12) we plot the shrinking factor (initial major axis divided by final major axis) for an initial condition with aspect ratio $=1.1$ and a final aspect ratio $=10$. Note how strong the dependence on $m$ is. Qualitatively speaking, that means that for large $m$, the hole will elongate only in the very final stage of the focusing, while for small $m$, the elongation starts to be evident in the initial stages. We conjecture that for $m \rightarrow \infty$ the shrinking factor tends to zero, indicating that the limiting hole never elongates, consistently with the Hele-Shaw limit.

\section{Asymptotic approximation}

We discuss here a simple approximation that gives a description which agrees very well with the numerical results presented above. Our demonstration is not rigorous, but is instead based in scaling arguments.

By using dimensional analysis, we can argue that the derivatives of $v$ in the $x$ direction scale as $v_{0} / x_{f}$ and that the derivatives in the $y$ direction scale as $v_{0} / y_{f}$. Since $x_{f}>>y_{f}$ asymptotically, the latter derivatives are dominant. Here $v_{0}$ is the typical scale of $v$ near the interface, for instance $v_{0}=v\left(0,2 y_{f}\right)$.

Thus we can use a boundary layer argument analogous to the one used in the Prandtl problem. We construct the asymptotic approximation by assuming that near the collapse, and for small distances to the focusing point, the terms of Eq. (3) that contain derivatives in the $x$ direction are negligible in comparison 
with the terms with derivatives in the $y$ variable:

$$
(m-1) v v_{x x}+v_{x}^{2}<<(m-1) v v_{y y}+v_{y}^{2} .
$$

Then our approximate solution is a collection of one-dimensional solutions $v_{0}(y, t)$ in the variable $y$ that satisfy

$$
v_{t}=(m-1) v v_{y y}+v_{y}^{2}
$$

with the variable $x$ being considered as a parameter or a constant of integration in each of these 1D solutions. Let us assume that all these solutions differ only by a delay of time $t_{0}(x)$

$$
v(x, y, t) \approx v_{0}\left(y, t-t_{0}(x)\right)
$$

Here, for convenience we are taking the focusing time to be $T=0$ so that $t<0$. Each portion of the interface will behave locally as a plane wave, so it is natural to choose the function $v_{0}$ to be the self-similar solution of two colliding plane waves that move at a constant velocity $V>0$ towards the origin,

$$
v_{0}(y, t)=V^{2} t W\left(\frac{|y|}{V t}\right)
$$

where

$$
W(\eta)= \begin{cases}\eta+1 & \eta \leq 0 \\ Q(\eta) & \eta>0\end{cases}
$$

and where $Q$ is the solution of the following ODE

$$
Q-\eta Q^{\prime}=(m-1) Q Q^{\prime \prime}+{Q^{\prime}}^{2} \quad Q^{\prime}(0)=0 \quad Q^{\prime}(\infty)=1
$$

In Fig. (13) we show a few members of this collection of $1 \mathrm{D}$ solutions for different values of $t$. Thus, from Eqs. (30) and (31) the asymptotic approximation may 
be written as

$$
v(x, y, t) \approx V^{2}\left(t-t_{0}(x)\right) W\left(\frac{|y|}{V\left(t-t_{0}(x)\right)}\right) .
$$

Finally, if we assume that the time-delay function $t_{0}(x)$ is twice differentiable and that has a local maximum, then its expression for small values of $x$ will be

$$
t_{0}(x) \approx-A x^{2}
$$

where $\mathrm{A}$ is a positive constant. Substituting in the previous expression for $v$ we obtain

$$
v(x, y, t) \approx-t V^{2}\left(X^{2}-1\right) W\left(\frac{|Y|}{X^{2}-1}\right)
$$

where the scaled distances $X$ and $Y$ are

$$
X=\frac{x}{\sqrt{-t / A}} \quad Y=\frac{y}{-V t}
$$

The interface of this expression is given by $\eta=\frac{|Y|}{X^{2}-1}=-1$. Here we are interested in the pre-focusing of the interface, for $t<0$. Thus, from Eq. (36) we can see that the solution has a universal structure.

In Fig. (10) we show the comparison between Eq. (36) and the numerical calculations of the previous section. In order to make these figures, we normalized the $x$ and $y$ dimensions with $x_{f}$ and $y_{f}$, respectively, and we divided Eq. (36) by $-t V^{2}$ and the numerical values by $v_{y}\left(0, y_{f}\right)$. We find that the approximation is good when the aspect ratio is of the order of 1000 or larger. 


\section{Formal asymptotics}

Here we will consider solutions $v$ to the porous medium pressure equation Eq.

(3) which are even in both the $x$ and $y$ variables,

$$
v(x, y, t)=v(-x, y, t)=v(x,-y, t)
$$

We will give a formal scaling argument which leads to the non-self-similar approximate focusing solution constructed in the previous section, and which indicates that this approximation is the appropriate local limit at the focusing time $T$. Just before the focusing time $T$ the interface is approximately given by

$$
|y|=T-t-x^{2}
$$

The construction presented here is not rigorous. However, starting from the formal solution presented here, it is likely that one can rigorously construct a true solution to the pressure equation Eq. (3) whose local asymptotic focusing behavior is described by the formal solution. The construction would use techniques analogous to those of [4].

We introduce the self-similar variables

$$
\xi=\frac{x}{T-t}, \eta=\frac{y}{T-t}, \tau=-\ln (T-t)
$$

and set

$$
v(x, y, t)=(T-t) V(\xi, \eta, \tau) .
$$

The function $V$ satisfies the equation

$$
\frac{\partial V}{\partial \tau}+\xi \frac{\partial V}{\partial \xi}+\eta \frac{\partial V}{\partial \eta}-V=M V \Delta V+|\nabla V|^{2}
$$


where $M=m-1$. This equation has plane waves

$$
V(\eta)=\lambda(\eta-\lambda)_{+}
$$

as steady states, and a colliding pair of these waves should describe an elongated focusing solution in the region where $x, y=O(T-t)$.

To extend this representation to a larger length scale we linearize around the plane wave, after first rescaling so that $\lambda=1$. Let

$$
V(\xi, \eta, \tau)=(\eta-1)_{+}+\varphi(\xi, \eta, \tau)
$$

Then $\varphi$ satisfies

$$
\frac{\partial \varphi}{\partial \tau}=L(\varphi)+\text { higher order terms }
$$

where

$$
L(\varphi)=M(\eta-1) \Delta \varphi-\xi \frac{\partial \varphi}{\partial \xi}+(2-\eta) \frac{\partial \varphi}{\partial \eta}+\varphi .
$$

The operator $L$ maps polynomials to polynomials and one has

$$
L\left(\xi^{k} \eta^{l}\right)=(1-k-l) \xi^{k} \eta^{l}+\text { lower order terms. }
$$

If one orders the basis $\left\{\xi^{k} \eta^{l}: k, l \geq 0\right\}$ by degree, then the matrix of $L$ is upper triangular, with diagonal entries

$$
\lambda_{k l}=1-k-l
$$

The $\lambda_{k l}$ are the eigenvalues of $L$, and each $\lambda_{k l}$ has an eigenfunction of the form

$$
\Phi_{k l}(\xi, \zeta)=\xi^{k} \zeta^{l}+\text { lower order terms }
$$

where we have replaced $\eta$ by the variable

$$
\zeta=\eta-1
$$


which vanishes on the free boundary of the plane wave. The perturbation $\varphi$ is given by

$$
\varphi(\xi, \eta, \tau)=\sum_{k, l \geq 0} A_{k l} e^{(1-k-l) \tau} \Phi_{k l}(\xi, \zeta),
$$

where the first few eigenvalues and eigenfunctions are given by

$$
\begin{array}{ll}
\lambda_{00}=+1 & \Phi_{00}=1 \\
\lambda_{10}=0 & \Phi_{10}=\xi \\
\lambda_{01}=0 & \Phi_{01}=\zeta-1 \\
\lambda_{20}=-1 & \Phi_{20}=\xi^{2}-2 M \zeta+M \\
\lambda_{11}=-1 & \Phi_{11}=\xi \zeta-\xi \\
\lambda_{02}=-1 & \Phi_{02}=\zeta^{2}-2(M+1) \zeta+M+1
\end{array}
$$

If we assume that $\varphi \rightarrow 0$ as $\tau \rightarrow \infty$, then $A_{00}=A_{10}=A_{01}=0$. By our assumption of reflection symmetry with respect to the $y$-axis, all of the eigenfuctions which appear in the expansion must be even functions of $\xi$. Therefore the expansion Eq. (51) has the form

$$
\varphi(\xi, \eta, \tau)=e^{-\tau}\left(A_{20} \Phi_{20}+A_{02} \Phi_{02}\right)+o\left(e^{-\tau}\right) .
$$

Add this to the plane wave $(\eta-1)_{+}=\zeta_{+}$to get

$$
V(\xi, \eta, \tau)=B e^{-\tau}+\left(1-2 e^{-\tau}\right) \zeta+A e^{-\tau} \xi^{2}+A_{02} e^{-\tau} \zeta^{2}+o\left(e^{-\tau}\right),
$$

where $A=A_{20}$ and $B=M A_{20}+(M+1) A_{02}$. We assume that arbitrary constant $A>0$ is fixed. The interface for Eq. (54) is given by

$$
\eta=1-e^{-\tau}\left(B+A \xi^{2}\right)+o\left(e^{-\tau}\right) .
$$


The form of Eq. (54) suggests that we use an anisotropic scaling with the new space variable

$$
\sigma=\xi e^{-\tau / 2}=\frac{x}{\sqrt{T-t}}
$$

The quantity $V$ as a function of $\sigma, \eta$, and $\tau$ satisfies

$$
\frac{\partial V}{\partial \tau}+\eta \frac{\partial V}{\partial \eta}+\frac{\sigma}{2} \frac{\partial V}{\partial \sigma}-V=M V \frac{\partial^{2} V}{\partial \eta^{2}}+\left(\frac{\partial V}{\partial \eta}\right)^{2}+e^{-\tau / 2}\left\{M V \frac{\partial^{2} V}{\partial \sigma^{2}}+\left(\frac{\partial V}{\partial \sigma}\right)^{2}\right\}
$$

We now look for a solution to Eq. (57) which matches Eq. (54) and which becomes stationary as $\tau \rightarrow \infty$.

$$
\begin{aligned}
& \text { If } V(\sigma, \eta, \tau) \rightarrow Z(\sigma, \eta) \text { as } \tau \rightarrow \infty \text {, then } Z \text { must satisfy } \\
& \qquad \frac{\sigma}{2} \frac{\partial Z}{\partial \sigma}=M V \frac{\partial^{2} Z}{\partial \eta^{2}}+\left(\frac{\partial Z}{\partial \eta}\right)^{2}-\eta \frac{\partial Z}{\partial \eta}+Z .
\end{aligned}
$$

For arbitrary $C>0$, we regard

$$
\theta=C-\sigma^{-2}=2 \int_{1 / \sqrt{C}}^{\sigma} \frac{d s}{s^{3}}
$$

as a time variable and substitute

$$
Z(\sigma, \eta)=\sigma^{2} z\left(\theta, \eta \sigma^{-2}\right)
$$

in Eq. (58) to obtain the one-dimensional porous medium equation

$$
\frac{\partial z}{\partial \theta}=M z \frac{\partial^{2} z}{\partial \rho^{2}}+\left(\frac{\partial z}{\partial \rho}\right)^{2}
$$

with $\rho=\eta \sigma^{-2}$.

For $|\sigma| \leq C^{-1 / 2}$ with arbitrary $C>0$,

$$
Z(\sigma, \eta)=\left(|\eta|-1+C \sigma^{2}\right)_{+}
$$


is a solution to Eq. (58). On the other hand, written as a function of the variables $\sigma, \eta$, and $\tau$, Eq. (54) becomes

$$
V(\sigma, \eta, \tau)=\eta-1+A \sigma^{2}+O\left\{\left(1+\eta^{2}\right) e^{-\tau}\right\}
$$

Thus if we choose $C=A$,

$$
V(\sigma, \eta, \tau) \rightarrow Z(\sigma, \eta) \text { as } \tau \rightarrow \infty
$$

Observe that

$$
z(\theta, \rho)=\sigma^{-2} Z\left(\sigma, \rho \sigma^{2}\right)=(|\rho|+\theta)_{+}
$$

is the plane wave solution to Eq. (61). The Graveleau solution to Eq. (61) is a pair of converging plane waves which meet at $\theta=0$. This solution can be continued in a self-similar way [AA2] for $\theta>0$ in the form

$$
z(\theta, \rho)=\theta Q\left(\frac{\rho}{\theta}\right)
$$

where $Q(\cdot)$ satisfies the ordinary differential equation

$$
Q(r)-r Q^{\prime}(r)=M Q(r) Q^{\prime \prime}(r)+\left\{Q^{\prime}(r)\right\}^{2} .
$$

In Appendix 3 we sketch a proof that $Q$ is convex, and that

$$
\frac{Q(r)}{\sqrt{1+r^{2}}}
$$

is uniformly bounded and bounded away from zero.

The self-similar continuation of $z$ for $\theta>0$ gives us the continuation of $Z$ for $\sigma>A^{-1 / 2}$

$$
Z(\sigma, \eta)=\left(A \sigma^{2}-1\right) Q\left(\frac{|\eta|}{A \sigma^{2}-1}\right)
$$


In the original variables the approximation $V \sim Z(\sigma, \eta)$ implies that

$$
\begin{gathered}
v(x, y, t)=(T-t) V\left(\frac{x}{\sqrt{T-t}}, \frac{y}{T-t},-\ln (T-t)\right) \sim \\
\left\{\begin{array}{l}
\left(|y|+A x^{2}+t-T\right)_{+} \text {for }|x| \leq \sqrt{\frac{T-t}{A}} \\
\left(A x^{2}+t-T\right) Q\left(\frac{y}{A x^{2}+t-T}\right) \text { for }|x| \geq \sqrt{\frac{T-t}{A}}
\end{array}\right.
\end{gathered}
$$

This is precisely the solution derived in the previous section. In view of the properties of $Q$ we conclude that

$$
\frac{v(x, y, t)}{\sqrt{\left(A x^{2}+t-T\right)^{2}+y^{2}}}
$$

is bounded both from above and below.

ACKNOWLEDGMENTS S. I. B. is grateful to the School of Mathematics of the University of Minnesota for a Visiting Position in 1999. We also thank the National Science Foundation, the Supercomputing Institute of the University of Minnesota, J. Hulshof for his results about the stability analysis for large $m$, and E. J. Doedel for the numerical values of the similarity exponent used in the validation of our computations.

\section{Appendix 1: ENO derivatives computation}

In order to compute the $|\nabla v|^{2}$ term, we use the ENO scheme, which has been used very succesfully in the numerical solution of Hamilton-Jacobi equations $[25$, 23]. The ENO scheme is an adaptive stencil interpolation procedure which automatically obtains information from the locally smoothest region, and hence yields a uniform high essentially nonoscillatory approximation for piecewise smooth functions. 
When computing the partial derivative respect to $x$ corresponding to $v_{j l}$ at discrete nodes $x_{j}=h j, j=0, \pm 1, \pm 2, \ldots$ we first write the undivided differences

$$
\begin{gathered}
w(j, 0)=v_{j l}, \\
w(j, k)=w(j+1, k-1)-w(j, k-1) \quad k=1, \ldots, r+1
\end{gathered}
$$

The ENO stencil-choosing procedure is optimally implemented by starting with $i(j)=j$ and performing

$$
\text { if } \quad|w(i(j), k)|>|w(i(j)-1, k)| \quad \text { then } \quad i(j) \leftarrow i(j)-1
$$

for $k=2, \ldots, r$. Finally we compute the forward and the backward derivatives,

$$
\left.(\partial v / \partial x)^{+}=\frac{1}{h} \sum_{k=1}^{r} c(i(j)-j), k\right) w(i(j), k)
$$

and

$$
\left.(\partial v / \partial x)^{-}=\frac{1}{h} \sum_{k=1}^{r} c(i(j-1)-j), k\right) w(i(j-1), k)
$$

where

$$
c(m, k)=\frac{1}{k !} \sum_{s=m}^{m+k-1} \prod_{l=m, l \neq s}^{m+k-1}(-l) .
$$

The same procedure is used to compute the derivatives in the $y$ direction. In order to compute the upwind approximation for $v_{x}^{2}$ we write

$$
v_{x}^{2}=\min \left((\partial v / \partial x)^{-}, 0\right)^{2}+\max \left((\partial v / \partial x)^{+}, 0\right)^{2}
$$

Similar expressions are used to compute $v_{y}^{2}$. This is known as the Osher-Sethian monotone Hamiltonian[26, 28], and it is very useful when there are discontinuities in the first derivatives, as in our case at the interface and for $m=1$. 


\section{$7 \quad$ Appendix 2: Stability analysis for large $m$}

Here we address the question of the stability of the self-similar solutions with circular symmetry for large $m$. In [11] a linear stability analysis is performed in terms of the radii of the contour lines of the solution in the self-similar plane, $r /(-t)^{\beta}=S(p, \theta, \tau)$, where $p=v(r, \theta, t)(T-t)^{1-2 \beta}$ is the scaled pressure, $\beta$ is the similarity exponent and $\tau=-\ln (-t)$. It is shown that the stability analysis may be reduced to a system of two ordinary differential equations

$$
n p \Psi \Psi^{\prime \prime}=n p\left(\Psi^{\prime}\right)^{2}-\beta\left(\Psi \Psi^{\prime}\right)^{2}+\Psi \Psi^{\prime}+(2 \beta-1) p \Psi\left(\Psi^{\prime}\right)^{3}
$$

and

$$
\begin{aligned}
n p \Psi^{2} A^{\prime \prime}+ & \left\{2 \beta \Psi^{\prime} \Psi^{3}-\Psi^{2}-2 n p \Psi \Psi^{\prime}-3(2 \beta-1) p\left(\Psi \Psi^{\prime}\right)^{2}\right\} A^{\prime} \\
& +\left\{n p\left(1-k^{2}\right)+(\beta-\lambda) \Psi^{2}\right\}\left(\Psi^{\prime}\right)^{2} A=0,
\end{aligned}
$$

subject to the initial and growth conditions at infinity

$$
\begin{array}{cc}
\Psi(0)=1 & \Psi(p) \sim p^{\beta /(2 \beta-1)} \text { for } p \gg 1, \\
A(0)=1 & A(p) \sim p^{(\beta-\lambda) /(2 \beta-1)} \text { for } p \gg 1,
\end{array}
$$

where $n=m-1,^{\prime}=d / d p, \Psi(p)=r /(-t)^{\beta}$ is the radius of the contour lines in the solution with circular symmetry as a function of the scaled pressure $p . A(p)$ represents the departure of the contour lines from circularity when the circulary symmetric solution is perturbed as $S(p, \theta, \tau)=\Psi(p)+A(p) \exp (i k \theta+\lambda \tau) . \quad k$ is the given wave number and $\lambda$ is an eigenvalue whose sign determines the stability (when $\lambda>0$, the solution is unstable), and its value must be determined numerically. 
For $k=2$, the effect of the perturbation is to elongate the shape of the hole. The stability analysis described in [11] was carried out only for a limited range of $m$-values. Here we shall extend the analysis to much larger values of $m$. In particular, we find that for $k=2$ the circularly symmetric self-similar solutions are unstable for $m$ as large as $10^{12}$.

a) Numerical integration of the ODE's: In order to integrate Eqs. (72) and (73), we use a Runge Kutta scheme of order 4 with adaptative step-size control (also known as Runge Kutta 4-5, described in detail in [27]) which allows to achieve a predetermined accuracy in the numerical solution. We set a relative tolerance of $10^{-12}$ and we implement the integration with quadruple precision (real $* 16$ Fortran variables).

b) Initial condition at $p=0$ : numerically, we cannot start integrating at $p=0$ because both equations are degenerate there. Thus, we start at $p=$ $10^{-20}$, and we compute the initial values of $\Psi$ and $A$ with a second order Taylor expansion of the solutions, which are given by

$$
\begin{gathered}
\Psi(p) \approx 1+\frac{1}{\beta} p+\frac{\beta(1+n)-1}{2(1+n) \beta^{3}} p^{2} \\
A(p) \approx 1+\frac{\lambda-\beta}{\beta^{2}} p+\frac{(\lambda-3) \lambda+\beta^{2}\left(n\left(k^{2}-3\right)-3\right)+\beta(3+2 \lambda(1+n))}{2(1+n) \beta^{4}} p^{2}
\end{gathered}
$$

c) Bracketing of the eigenvalue $\beta$ : According to Eq. (74), the function $\Psi(p)$ must grow algebraically for large $p$. However, if we have the wrong value of the 
eigenvalue $\beta, \Psi$ will either diverge or go to zero. In order to find the eigenvalue, we start integrating with a guess value of $\beta=1 / 2$ and an increment $\Delta \beta=1 / 4$, and if $\Psi \rightarrow \infty$ we set $\beta=\beta-\Delta \beta$ and when $\Psi \rightarrow 0$ we set $\beta=\beta+\Delta \beta$. Then we divide the value of $\Delta \beta$ by a half. Iterating this procedure yields increasingly better approximations for the eigenvalue $\beta$. The algorithm converges typically after 60 cycles. We checked the accuracy of this computation by comparing with the values obtained by E. J. Doedel using AUTO [14]. We also compared the values with an asymptotic approximation for $\beta$ derived by J. Hulshof [18].

d) Bracketing of the eigenvalue $\lambda$ : Once the solution $\Psi$ is computed, we apply a similar iterative scheme to find $\lambda$. According to the analysis presented in [11] $A(p)$ may grow either algebraically or exponentially. We can detect this numerically using Eq. (74) as an estimate for $p>1$ (if $\lambda$ is larger than the eigenvalue, $A(p)$ will be orders of magnitude larger than the estimate, and if $\lambda$ is smaller, we obtain negative values with absolute values of orders of magnitude larger than the estimate). Then iterate to bracket the value of $\lambda$.

With this method we reproduced the results reported in [11] for $m \leq 2$. In Fig. (14) we show the values obtained for $\lambda$ as a function of $m-1$. The values are all positive indicating that the solution is unstable respect to elongations on the initial shape of the hole. 


\section{Appendix 3: properties of $Q$}

In this Appendix we sketch proofs of the properties of the function $Q$ cited in Section 5 .

- $Q$ is a convex function.

Formally we differentiate Eq. (61) twice with respect to $\rho$ to get

$$
\frac{\partial w}{\partial \theta}=M z \frac{\partial^{2} w}{\partial \rho^{2}}+(2 M+2) \frac{\partial z}{\partial \rho} \frac{\partial w}{\partial \rho}+(M+2) w^{2}
$$

where $w=\partial^{2} z / \partial \rho^{2}$. For $\theta=0$ we have $z=|\rho|$ so that $w=0$. The maximum principle then gives us $w>0$ for $\theta>0$. More precisely, $z$ is obtained as the limit as $\varepsilon \searrow 0$ of the solution $z^{\varepsilon}$ to Eq. (61) with initial value $z_{0}^{\varepsilon}(\rho)=\sqrt{\varepsilon^{2}+\rho^{2}}$. The solution $z^{\varepsilon}(\rho, \theta)$ is smooth since it is everywhere positive. Thus one can apply the above reasoning to $z^{\varepsilon}$ instead of $z$, and conclude that the $z^{\varepsilon}$ are all strictly convex. The convexity of $z$ follows by letting $\varepsilon \searrow 0$.

- Behaviour for large $r$.

$$
Q(r)=r+1+O\left(e^{-\delta r}\right)
$$

for some constant $\delta>0$. This expansion can be differentiated any number of times with respect to $r$.

$$
z(\theta, \rho)=\theta Q(\rho / \theta) \text { is a solution to Eq. (61). Near } \rho=1 \text { it is strictly }
$$
positive so that Eq. (61) is nondegenerate. The initial values of $z$ for $\rho>0$ are $z(\rho, 0)=\rho$, and a solution to Eq. (61) with these initial values is $z^{*}(\theta, \rho)=\rho+\theta$. Near $\rho=1$ for small $\theta \geq 0$ the difference $w=z-z^{*}$ is then the solution to the 
linear parabolic equation

$$
\frac{\partial w}{\partial t}=M z w_{\rho \rho}+\left(\frac{\partial z}{\partial \rho}+1\right) \frac{\partial w}{\partial \rho}
$$

with initial data $w(\rho, 0)=0$. For $\rho$ close to 1 and $\theta$ close to 0 , both $z$ and $\partial z / \partial \rho$ are close to their initial values so that the coefficients in Eq. (76) are bounded. In a domain $[1-\nu, 1+\nu] \times\left[0, \theta_{0}\right]$ the solution to the boundary initial value problem for Eq. (76) is given by integrals over the lateral sides of the domain involving $w$ and the Green function. If $\theta_{0}$ is sufficiently small, then we can conclude from standard properties of the Green function that $w(1, \theta)=O\left(e^{-\delta / \theta}\right)$ for some constant $\delta>0$. This asymptotic expansion can be differentiated infinitely often. To get the expression for $Q$ we set $r=1 / \theta$ and write

$$
Q(r)=\frac{1}{\theta} z(\theta, 1)=\frac{1}{\theta}\left\{z^{*}(\theta, 1)-z(\theta, 1)\right\}=1+r+O\left(e^{-\delta r}\right) .
$$

As a consequence we can derive Eq. 68 .

\section{References}

[1] Angenent S. B. and Aronson D. G., "Intermediate asymptotics for convergent viscous gravity currents" Phys. Fluids 7, 223 (1995).

[2] Angenent S. B. and Aronson D. G., "The focusing problem for the radially symmetric porous medium equation" Comm. P.D.E. 20, 1217 (1996).

[3] Angenent S. B. and Aronson D. G., "Self-similar non-radial hole filling for the porous medium equation", preprint (1999). 
[4] Angenent S. B. and Velazquez J. J. L., "Degenerate neckpinches in mean curvature flow", J. Reine Angew. Math. 482 (1997), 15-66.

[5] Aronson D. G., "The porous medium equation. In Some problems in Nonlinear Diffusion" (eds. A. Fasano and M. Primicerio), Lecture Notes in Math. No. 1224, Springer-Verlag (1986).

[6] Aronson D. G. and Graveleau J. "A self-similar solution to the focusing problem for the porous medium equation", Euro. Jnl. of Appl. Math. 4, 65 (1993).

[7] Aronson D. G., Gill O. and Vasquez J. L., "Limit behaviour of focusing solutions to nonlinear diffusions", Comm. PDE, 23 307-332 (1998)

[8] Barenblatt G. I. "On some unsteady motions of fluids and gases in a porous medium", Prikl. Mat. Mekh. 16, 67 (1952).

[9] Barenblatt G. I. "Scaling, Self-Similarity and Intermediate Asymptotics", Cambridge University Press, (1996).

[10] Berger M. and Kohn R., "A rescaling algorithm for the numerical calculation of blowing-up solutions", Comm Pure and Appl Math, 41, pp. 841-863 (1988).

[11] Betelu S. I., Aronson D. G and Angenent S. B. "Renormalization study of two-dimensional convergent solutions of the porous medium equation", Physica D 138 pp. 344-359 (2000) 
[12] Diez J., Gratton R., and Gratton J., "Self-similar solution of the second kind for a convergent viscous gravity current", Phys. of Fluids A 4 p. 1148 (1992)

[13] Diez J., Thomas L. P., Betelu S., Gratton R., Marino B., Gratton J., Aronson D. G., and Angenent S. B., "Noncircular converging flows in viscous gravity currents", Phys. Rev. E 58 p. 6182-6187 (1998)

[14] Doedel E. J., private communication.

[15] Chen L. Y. and Goldenfeld N., "Numerical renormalization-group for similarity solutions and travelling waves", Phys. Rev. E, 51 p. 5

[16] Goldenfeld N., "Lectures on phase transitions and the renormalization group", Addison-Wesley, Boston, 1992, p. 326.

[17] Gratton, J. and Minotti, F. (1990) "Self-similar viscous gravity currents: phase-plane formalism ", J. Fluid. Mech., 210, 155.

[18] Hulshof J., private communication.

[19] Huppert H. E. (1982) "The propagation of two dimensional viscous gravity currents over a rigid horizontal surface ", J. Fluid Mech., 121, 43.

[20] Lions P. L., Souganidis P. E., and Vazquez J. L., "The relation between the porous medium equation and the Eikonal equation in several space dimensions", Rev. Mat. Iberoamericana, 3(1987), 275-310.

[21] Murray J. D., "Mathematical Biology", Second corrected edition, Springer, Berlin, p. 238 (1993). 
[22] Sethian J. A., Level Set Methods: Evolving Interfaces in Geometry, Fluid Mechanics, Computer Vision and Material Science, Cambridge University Press, Cambridge, 1996

[23] Shu C. W. and Osher S., "Efficient implementation of essentially nonoscillatory shock capturing schemes", II, J. Comp. Phys, 83 p. 32-78 (1988).

[24] Sussman M, Smereka P and Osher S, "A level-set approach for computing solutions to incompressible two-phase flow", J. Comp. Phys. 114 pp. 146159 (1994).

[25] Osher S. and Shu C. W., "High-order essentially nonoscillatory schemes for hamilton jacobi equations", Siam J. Numer. Anal., 28, p. 907-922 (1991).

[26] Osher S. and Sethian J. "Fronts propagating with curvature dependent speed: algoritms based in Hamilton-Jacobi formulation", J. Comput. Phys. 79, (1988) pp. 12-49

[27] Press, W., Teukolsky, S., Vatterling, W. and Flannery, B., "Numerical Recipes: The Art of Scientific Computing", Cambridge University Press, (1992).

[28] Chi-Wang Shu, "Essentially non-oscillatory and weighted essentially nonoscillatory schemes for hyperbolic conservation laws", Advanced numerical approximation of nonlinear hyperbolic equations (Cetraro, 1997), 325-432, Lecture Notes in Math., 1697, Springer, Berlin, 1998. 


\section{LIST OF CAPTIONS}

1. Sketch of the focusing problem: fluid sorrounds a closed empty cavity. Then the fluid diffuses inwards and fills the cavity. We are interested in problems with mirror symmetries about the $x$ and $y$ axis. The function $\phi$ describes the shape of the interface.

2. Near the interface, all the derivatives of $v$ are computed using the points of behind. Thus, the approximations of the derivatives do not contain points from inside the hole. On the contrary, for the derivatives of $\phi$ inside the hole, we may use points across the interface.

3. The pressure $v$ (full line) does not grow when $\phi_{i j}<0$ ( $v_{i j}$ in right profile). Thus $v_{i j}$ is updated only when the interface crosses over the point $\left(x_{i}, y_{j}\right)$. By properly reducing the time-step we can capture accurately the moment when the interface passes exactly over this point (left profile), and only after this moment we start to update $v_{i j}$ with the Euler scheme.

4. The shape of the computation grid follows the shape of the interface. When the aspect ratio of the interface $x_{f} / y_{f}$ increases by a factor two, the $L_{y}$ dimension of the grid is divided in two, maintaining the same amount of gridpoints.

5. Contour lines of the explicit solution for $m=1$ for $t=-1$. The inner contour line is the interface $v=0$. The major and minor axis of the interface close with exponents exactly equal to $1 / 2$ and 1 , respectively.

6. Cumulative error as a function of the number of gridpoints, for $m=1$. 
7. Contour plots of the pressure $v$ for $m=2$ and various times approaching the focusing. The first plot is the initial condition with elliptical interfaces, with an aspect ratio of 1.1. In the second, the major axis has shrunk by a factor 4 , the third by a factor 16 and in the fourth by a factor 64 . In this simulation we did not regrid in the $y$-direction in order to show the increase in the aspect ratio of the interface. We renormalized isotropically the $x$ and $y$ coordinates by the same factor.

8. Contour plots of the pressure $v$ for $m=2$ and various times approaching the focusing. The $x$ and $y$ coordinates were rescaled with $x_{f}$ and $y_{f}$, respectively. The first plot is the initial condition with elliptical interfaces, with an aspect ratio of 1.1. In the second plot, the number of renormalizations, $y$-divisions and aspect ratio are, respectively, $(2,0,1.5)$, in the third, $(4,1,2.66)$ and in the fourth, $(7,4,20.6)$.

9. Contour plots of the pressure $v$ for $m=2$, with initial rectangular equispaced contour lines as the initial condition. The first plot is the initial condition, with an aspect ratio of 1.1. In the second plot, the number of renormalizations, $y$-divisions and aspect ratio are, respectively, $(2,0,1.4)$, in the third, $(4,1,2.17)$ and in the fourth, $(7,4,27.7)$.

10. Comparison of the contour lines of the numerical solution (lines) with the asymptotic approximation (dots), for $m=2$ (above) and $m=4$ (below). The aspect ratio is close to 1000 in both cases.

11. Major (hollow points) and minor (full points) axis dimensions of the in- 
terface, as a function of the time. Asymptotically, they are power laws of exponents $\approx 1 / 2$ and $\approx 1$ (full lines), for $m=2$ (diamonds) and $m=4$ (circles). Note how many orders of magnitude in the time scale we can describe with this numerical procedure.

12. Shrinking factor $x_{f}(t) / x_{f}(0)$ for which the aspect ratio goes from $x_{f} / y_{f}=$ 1.1 to 10. The larger the value of $m$ the smaller the hole has to be in order to observe the asymptotic behavior.

13. Collision of two plane waves that move towards the origin, for $m=2$. We show a few profiles of $v(y)$ before and after the collision time $(t=0)$.

14. Eigenvalues $\lambda$ as a function of the nonlinearity exponent $m$. The positive values mean that the mode $k=2$ is unstable in this interval. 



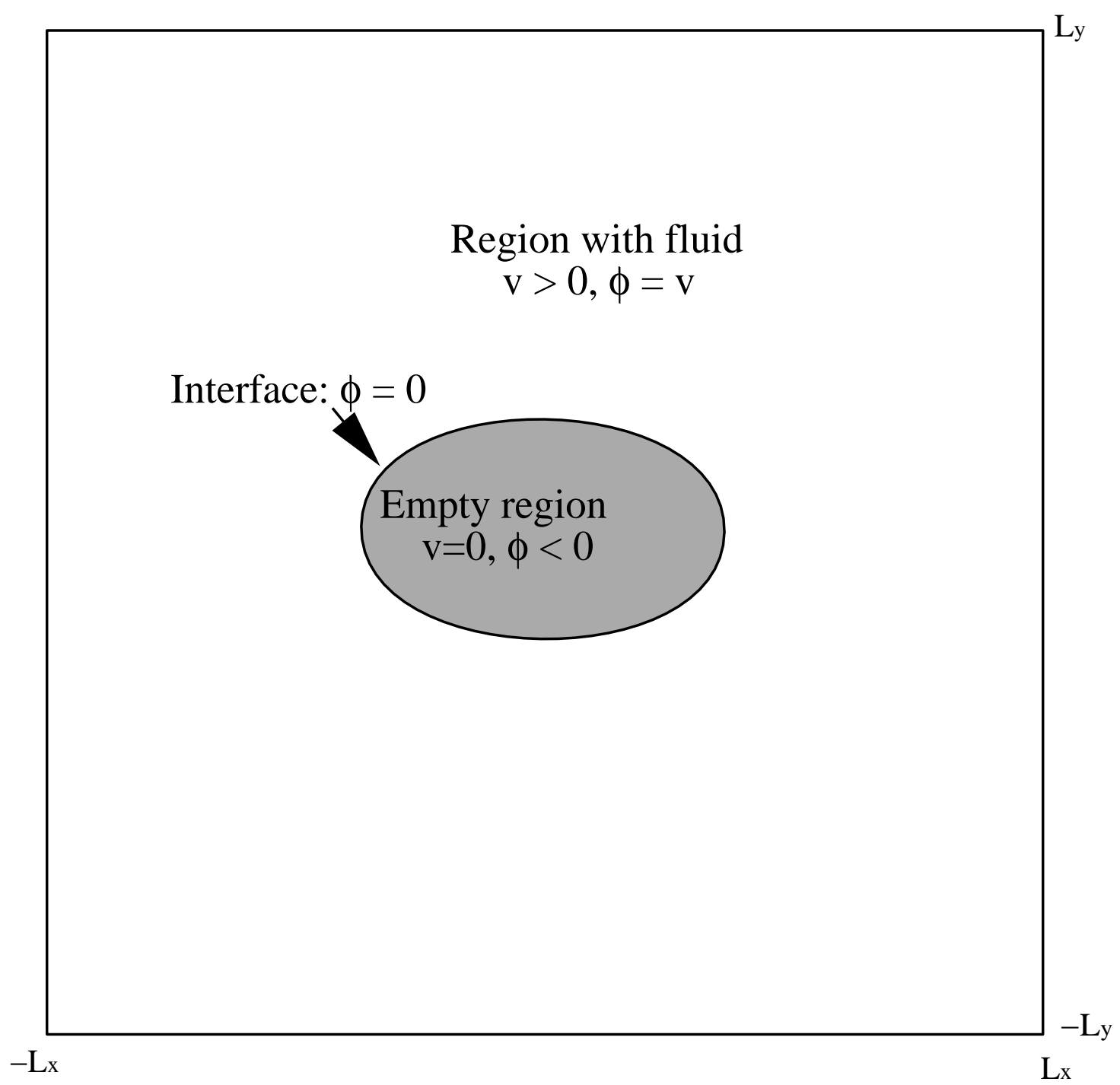

Figure 1: 


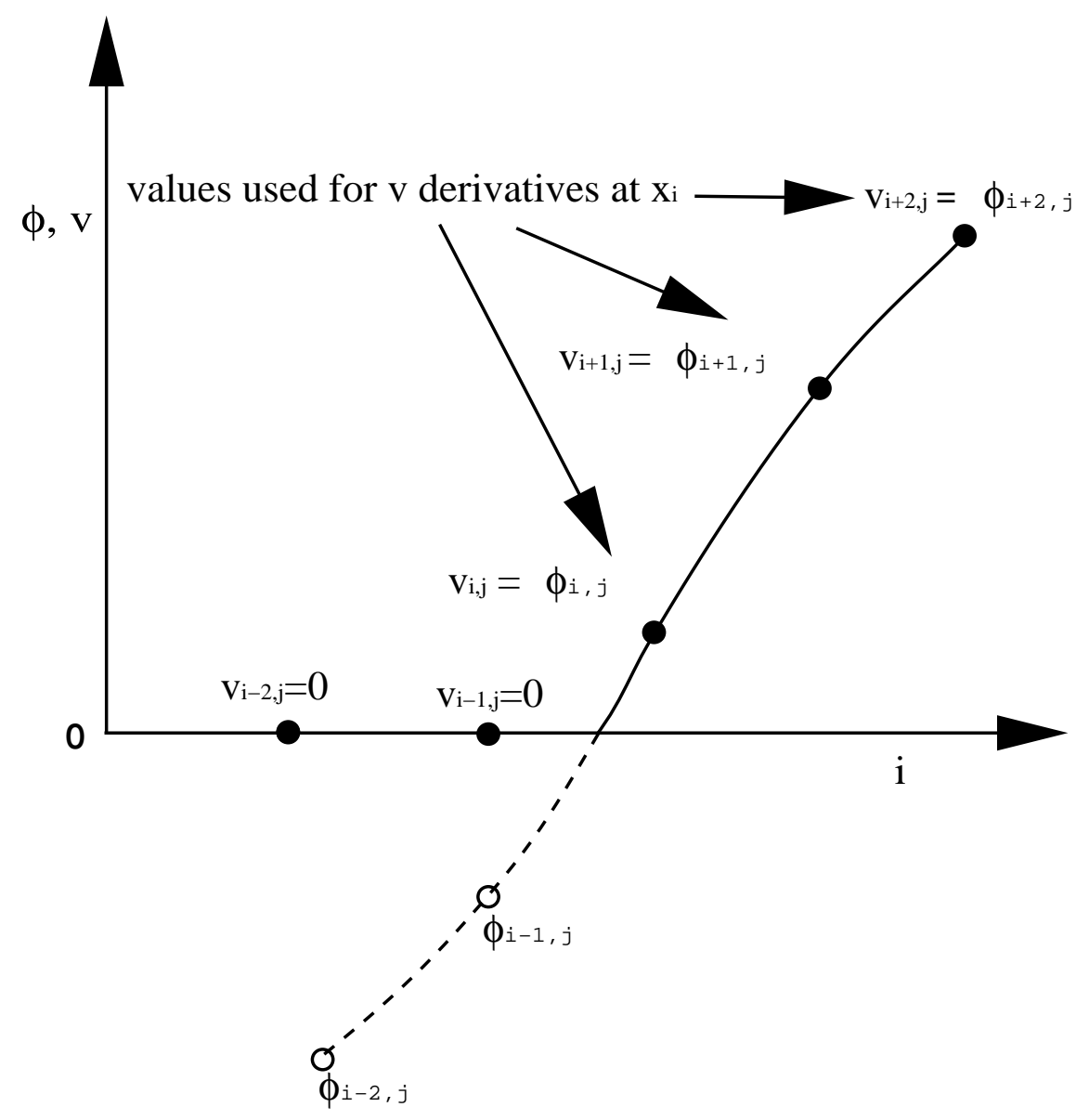

Figure 2: 


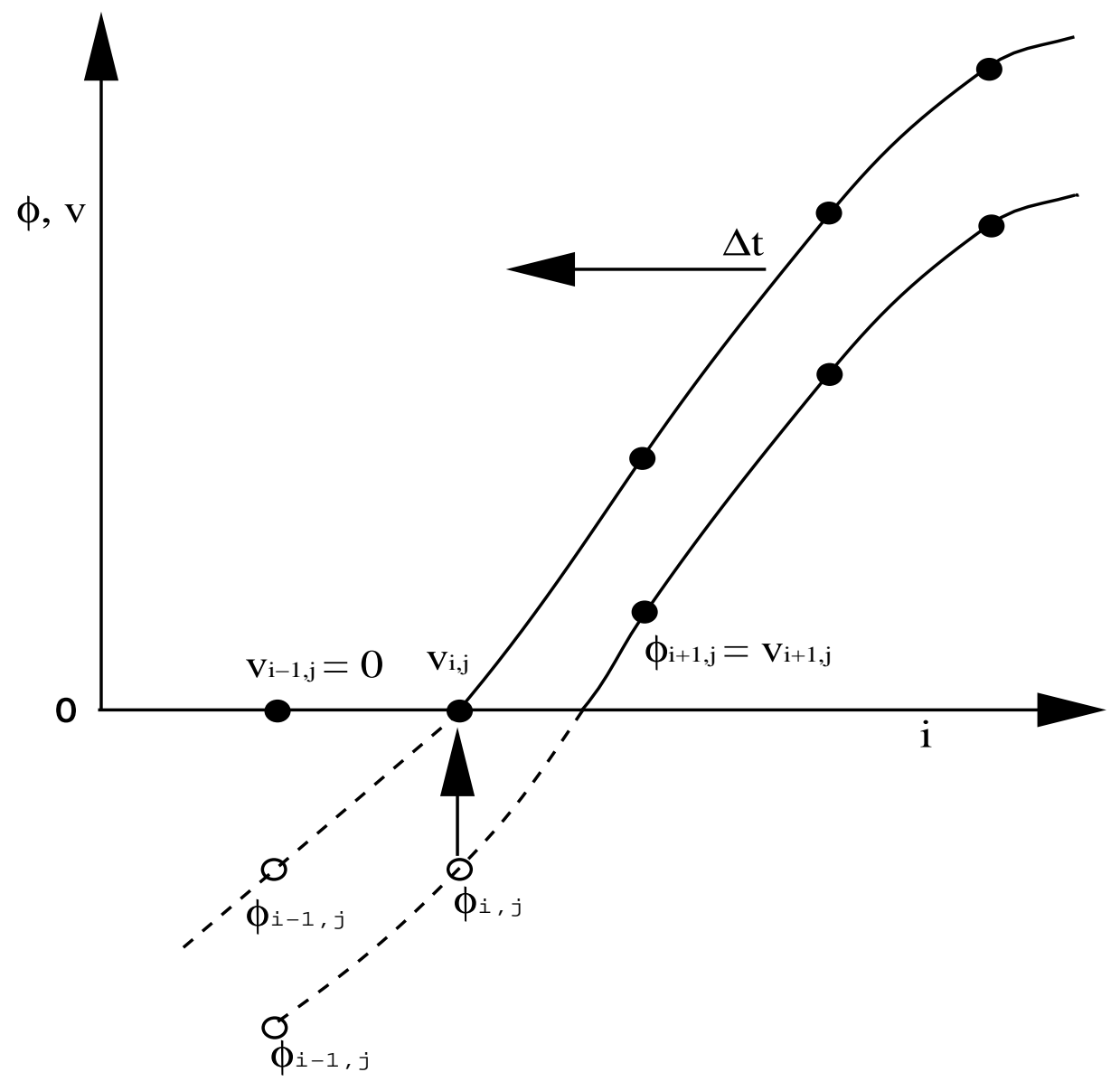

Figure 3: 


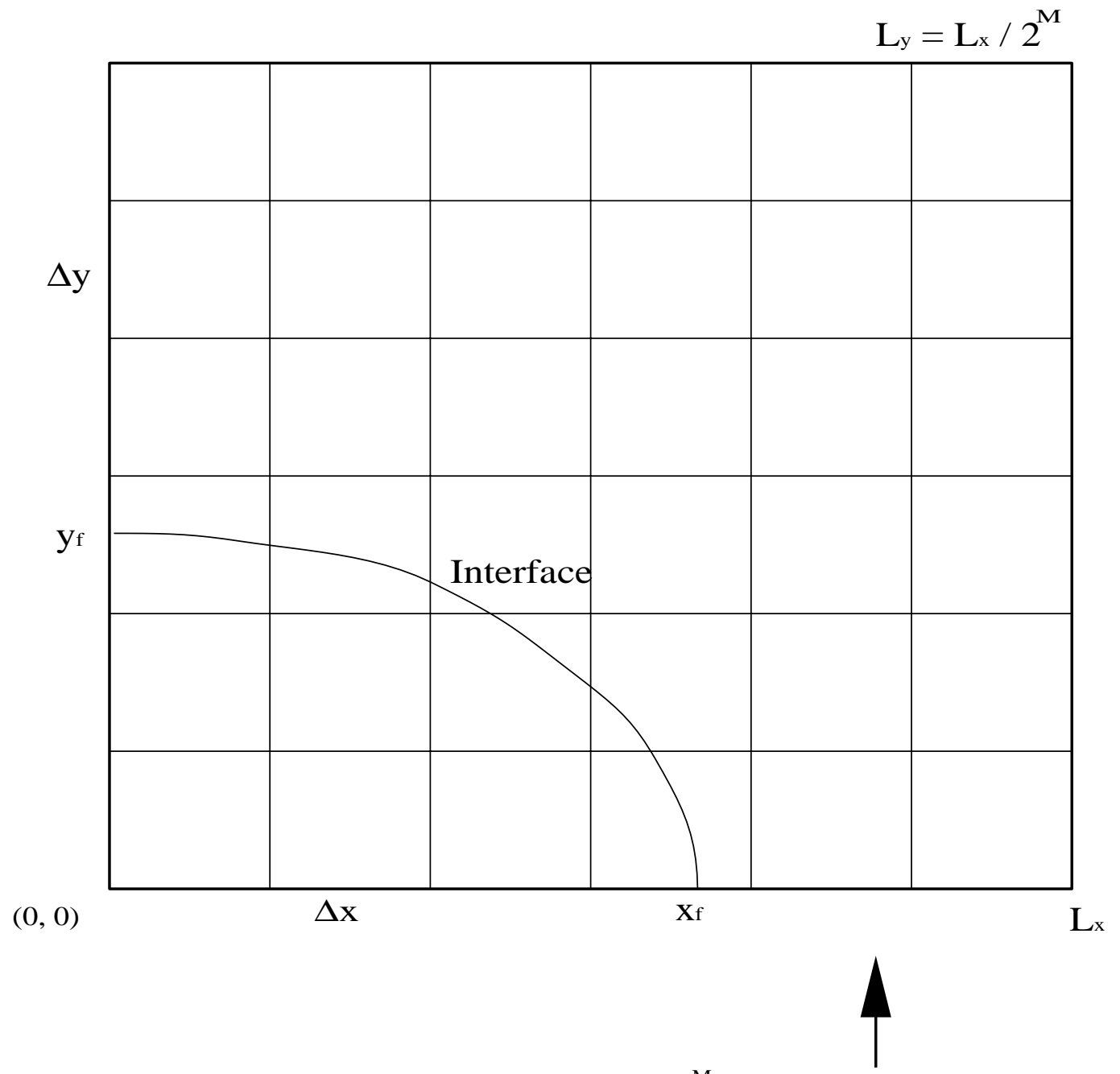

Actual aspect ratio of grid $=2^{\mathrm{M}}$

\begin{tabular}{|l|l|l|l|l|l|}
\hline & & & & & \\
\hline & & & & & \\
\hline & & & & & \\
\hline & & & & & \\
\hline & & & & & \\
\hline
\end{tabular}

Figure 4: 


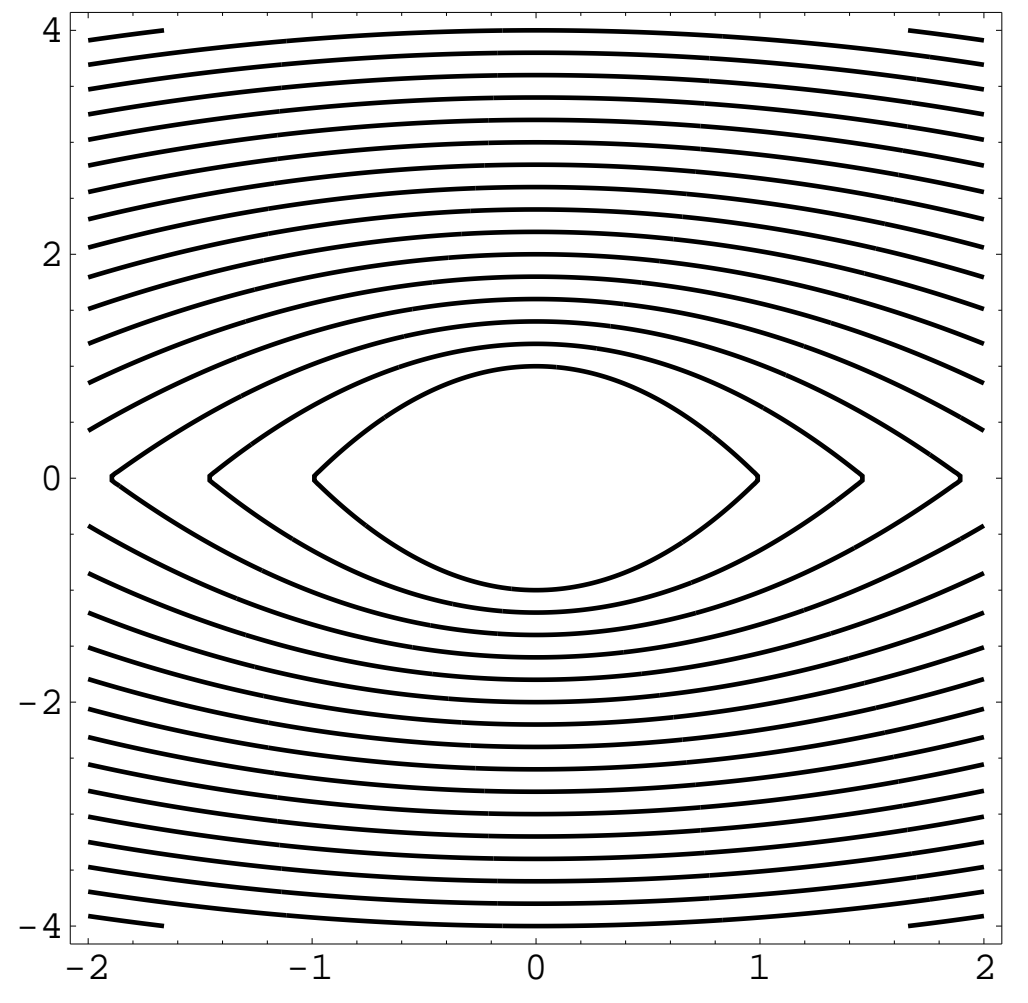

Figure 5: 


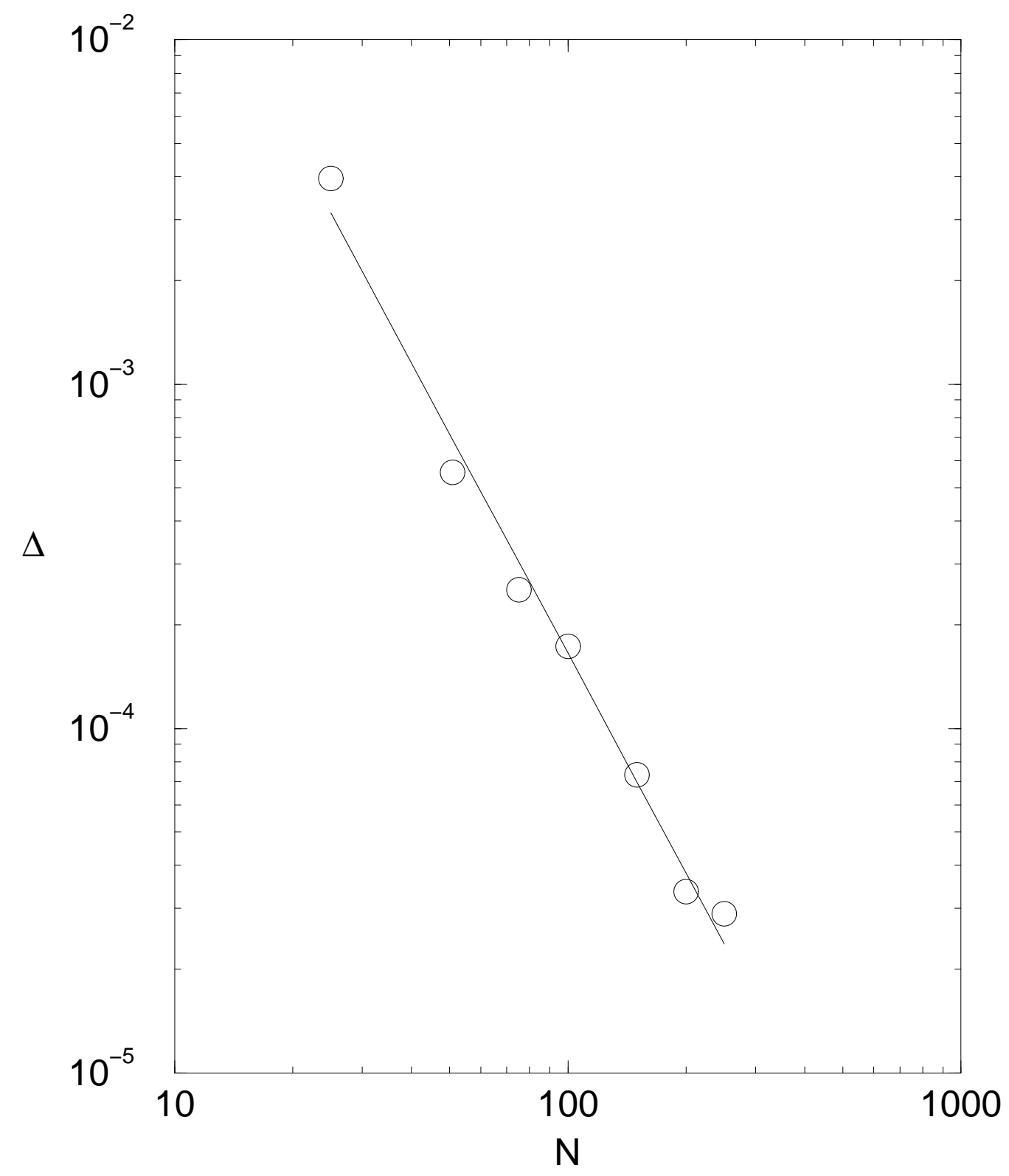

Figure 6: 

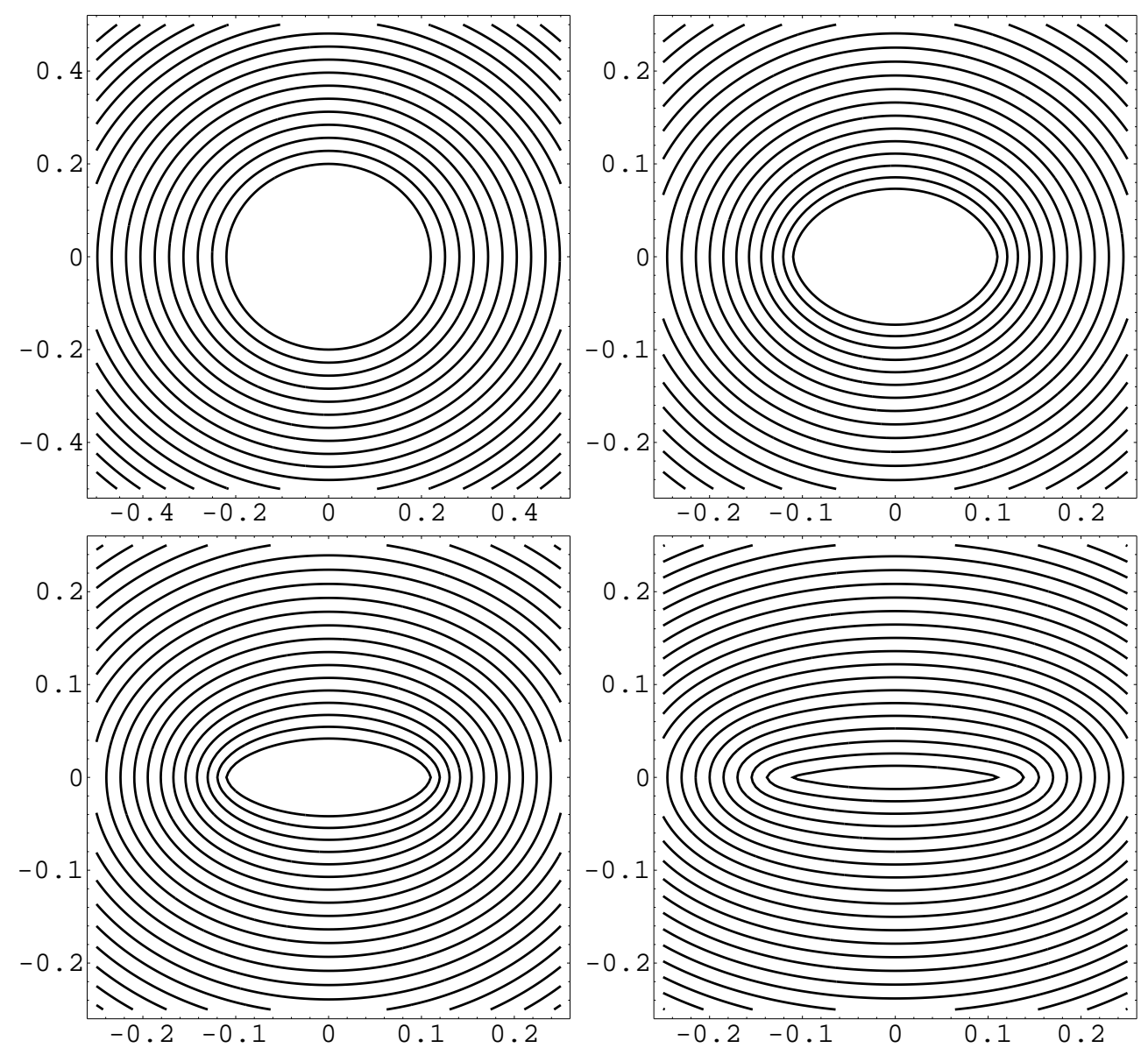

Figure 7: 

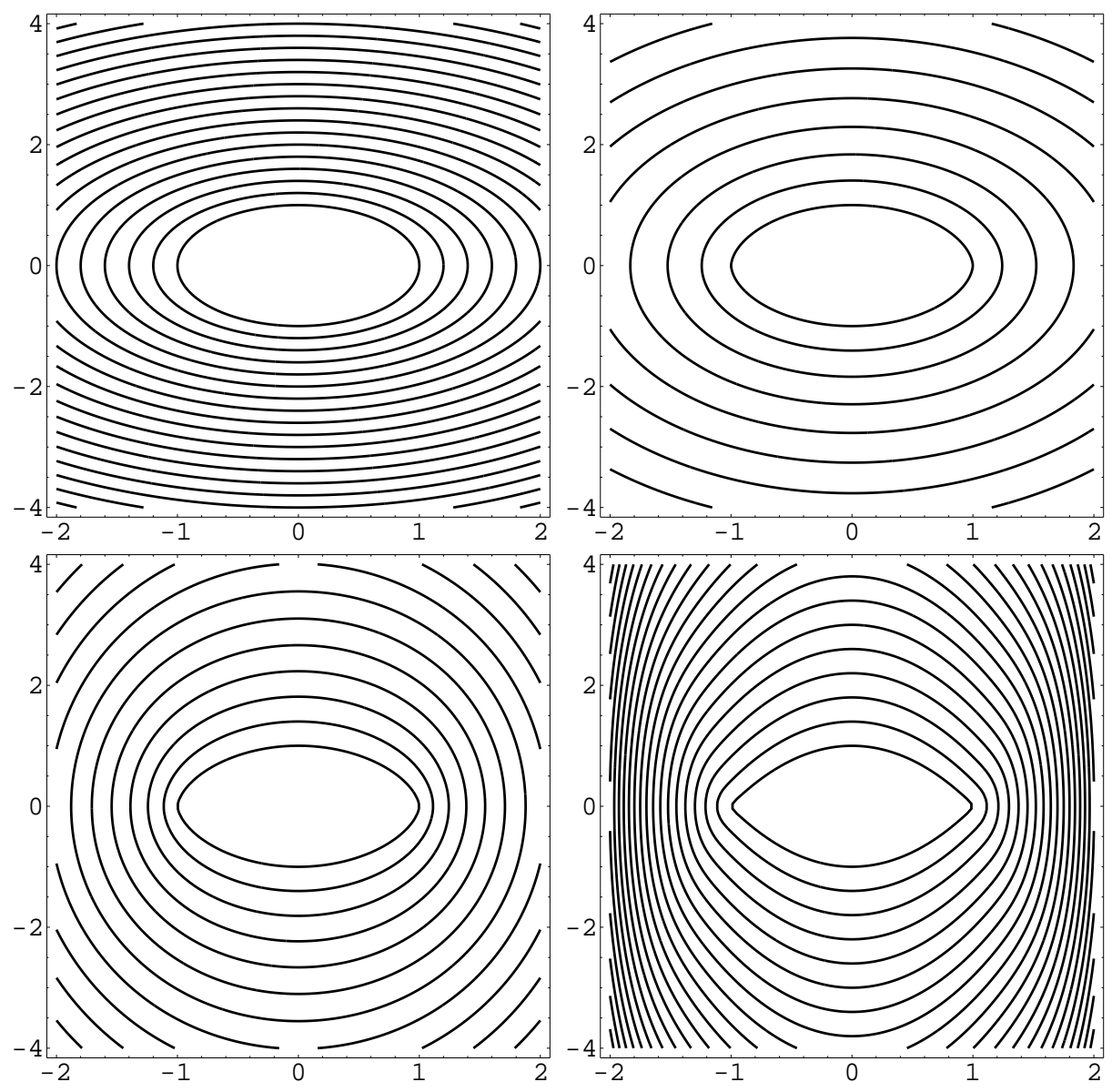

Figure 8: 

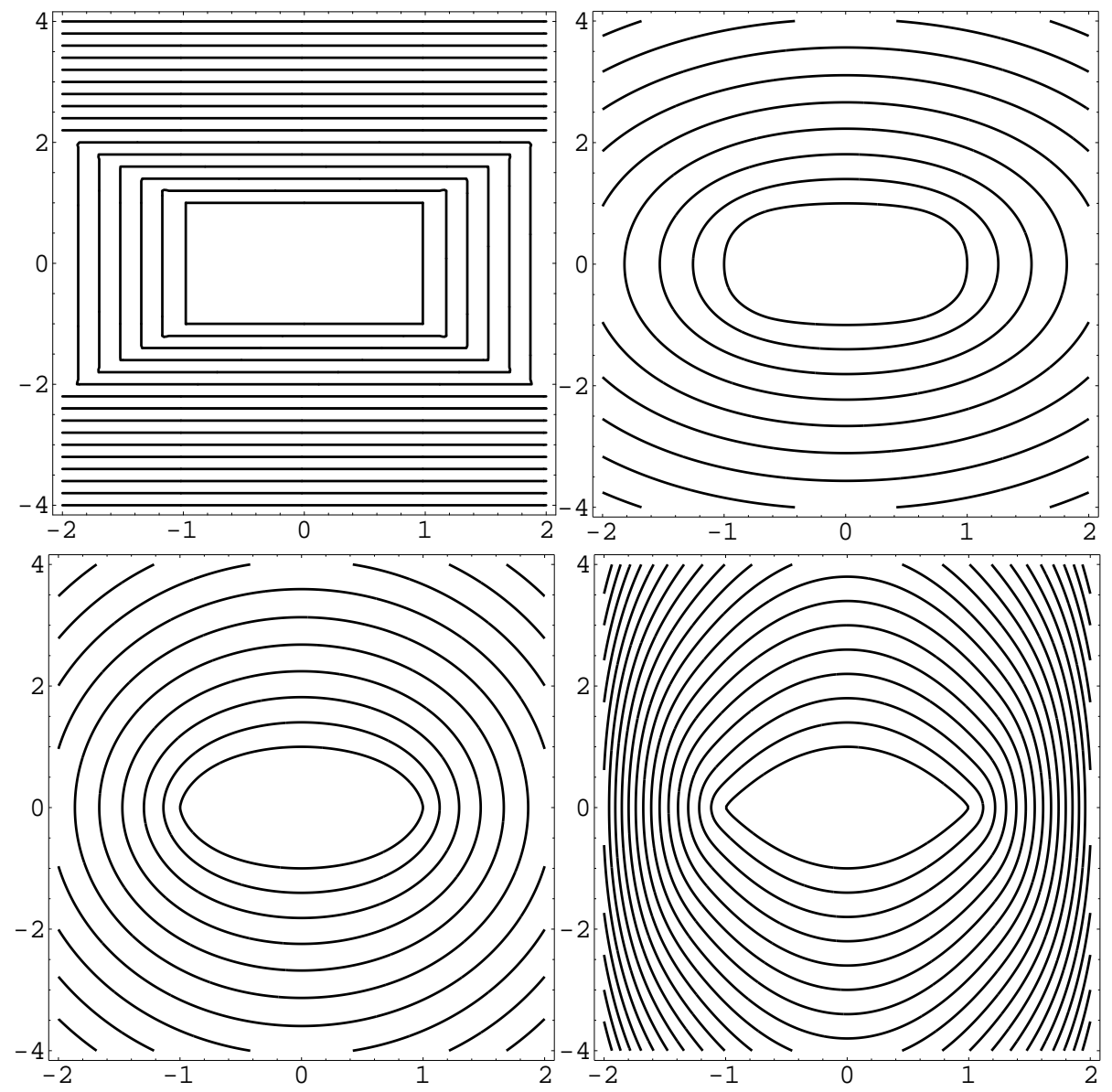

Figure 9: 

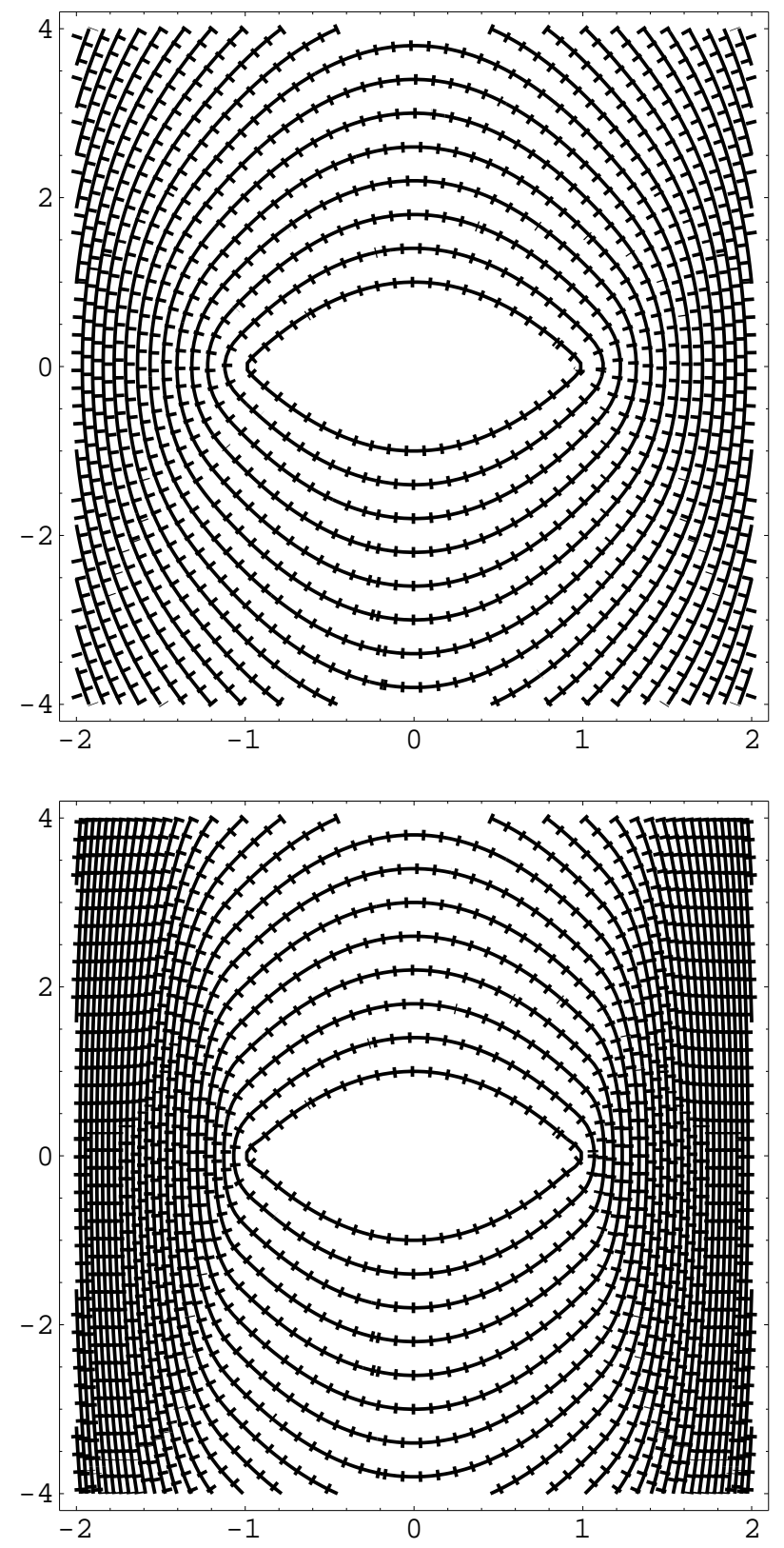

Figure 10: 


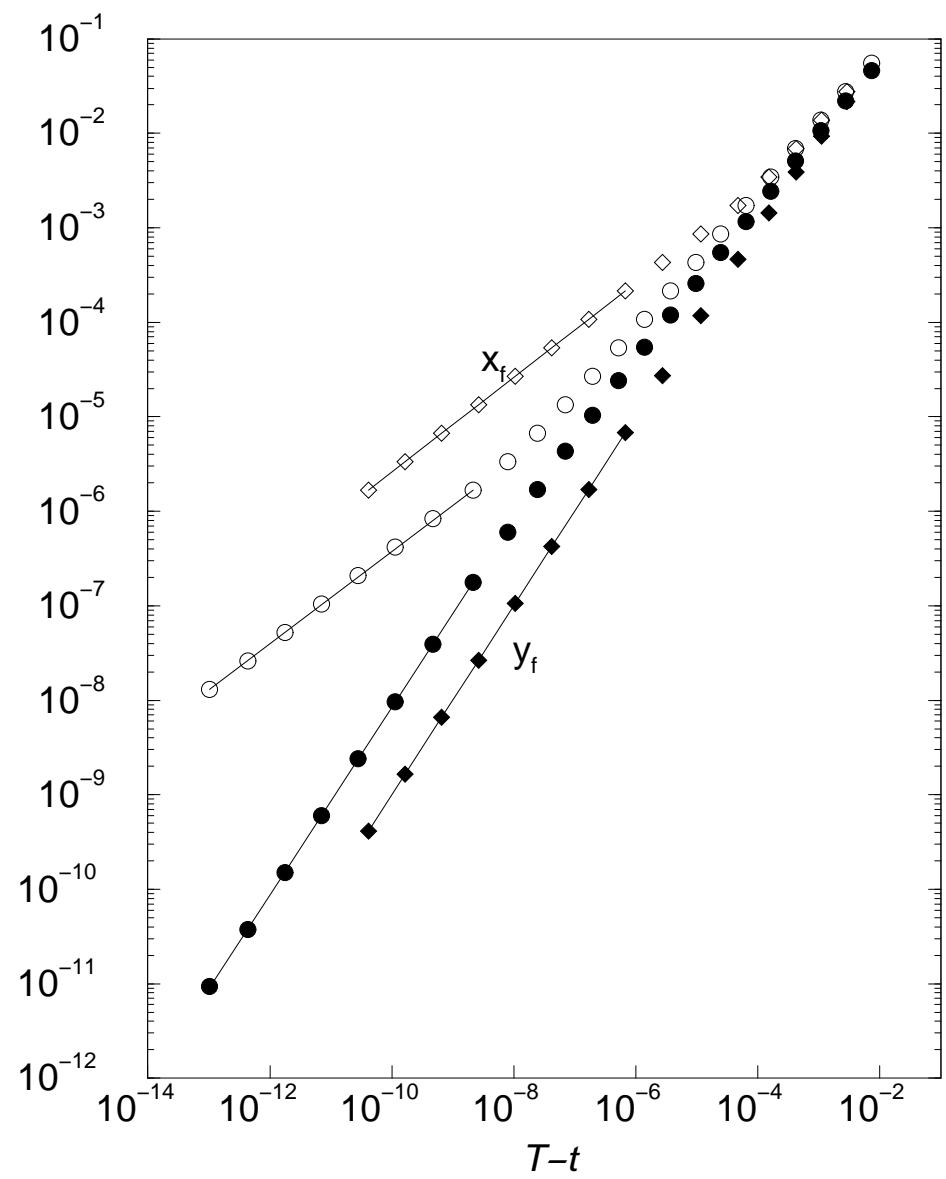

Figure 11: 


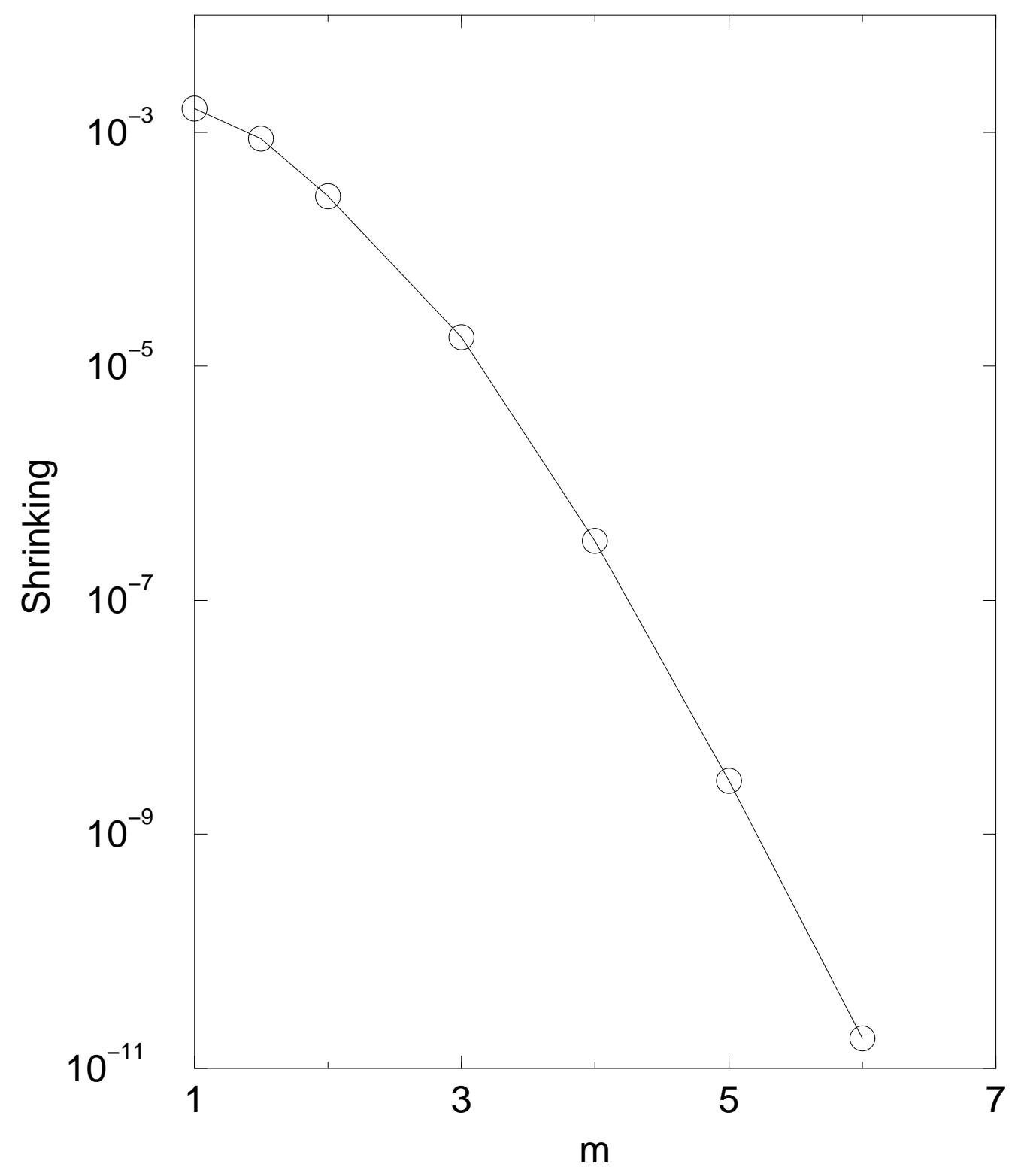

Figure 12: 


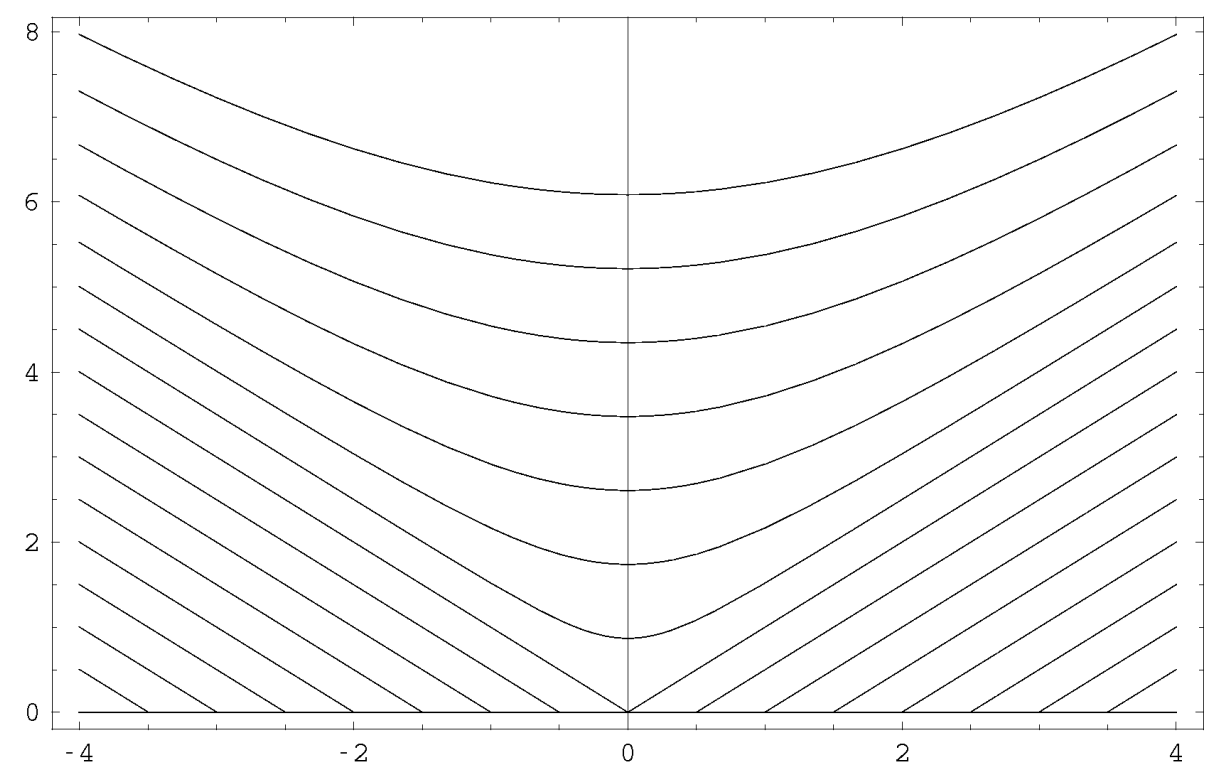

Figure 13: 


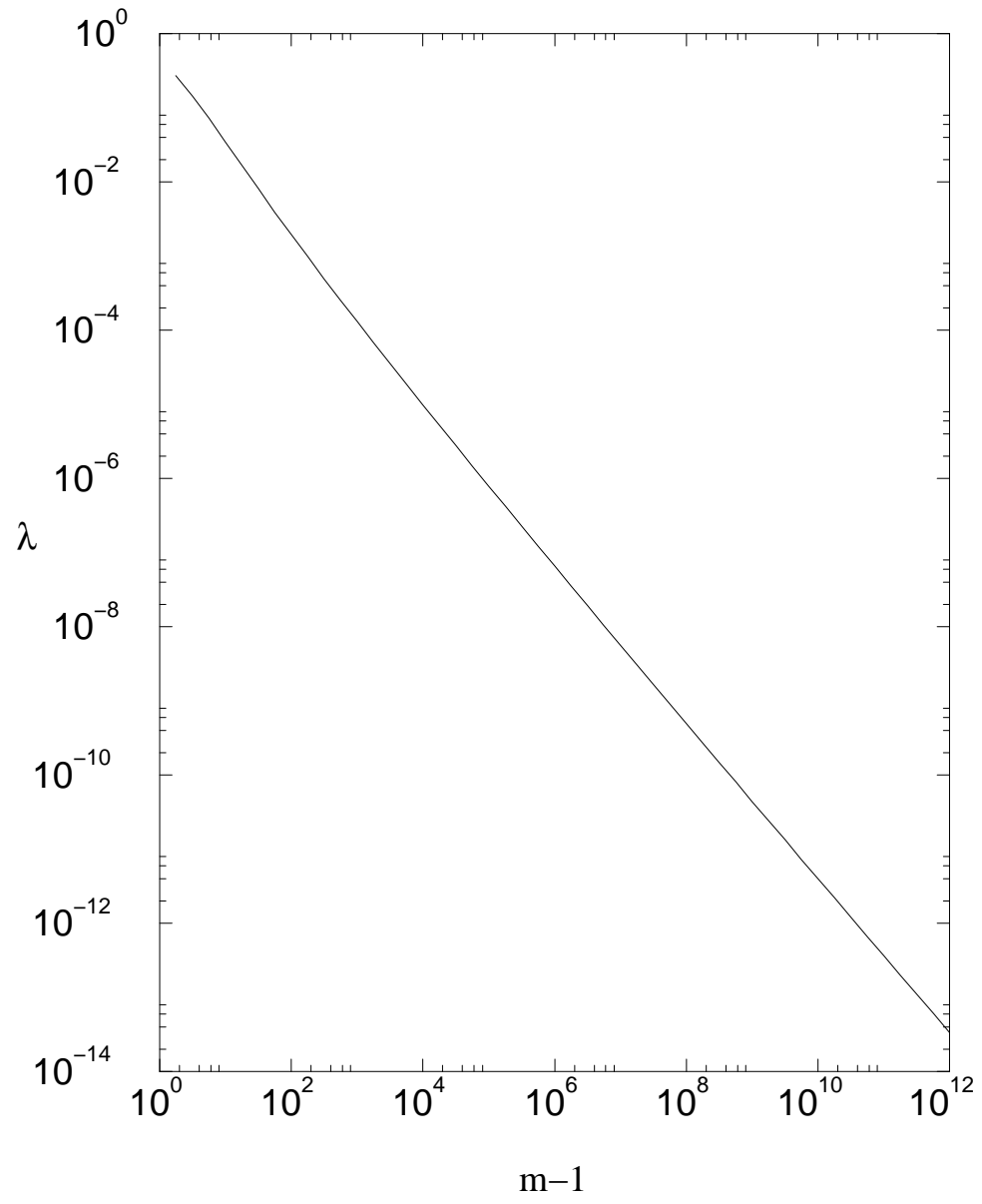

Figure 14: 